\title{
ANALYTIC FUNCTIONS AND THE FOURIER TRANSFORM OF DISTRIBUTIONS, II $\left.{ }^{1}\right)$
}

\author{
BY \\ LEON EHRENPREIS
}

1. Introduction. The present paper is a continuation of the work of Part I (see [5]) in which we showed how to define the Fourier transform of an arbitrary distribution of L. Schwartz. We were led, in a natural manner, to the consideration of linear functions on spaces of analytic functions. In $\$ 2$, below, we shall show how our previous methods apply also when dealing with indefinitely differentiable maps on Euclidean space of compact carrier with values in an arbitrary topological vector space $V$. The Fourier transform of such a map is an "entire map of exponential type" of the Euclidean space into $V$, and any such entire map of exponential type is the Fourier transform of an entire map of compact carrier of the Euclidean space into $V$. Once this generalized Paley-Wiener theorem is established, all our previous method on the Fourier transform of distributions can be extended to this generalized situation.

In this connection we should mention that there are two methods of defining vector-valued distributions; the general kernel theorem of $\mathrm{L}$. Schwartz states that these two methods yield the same result. We present in $\$ 2$ a simple proof of this theorem.

We consider also in $\$ 2$ the concepts of an analytic function on a topological vector space, and, more generally, of an analytic map of one topological vector space into another. Our notion of analytic map is a generalization of analyticity for Banach spaces (see [6, Chapter 4]). We expect to use analytic functions on the space $\mathfrak{D}$ (see $[5 ; 7]$ ) to extend the theory of distributions of L. Schwartz so as to apply to certain kinds of nonlinear differential equations. In certain cases we can prove that our analytic functions are limits of polynomials (i.e. belong to the ring generated by constants and linear functions). This situation is markedly different from that for infinite dimensional Banach spaces.

In $\S 3$ below, we consider some applications of the Fourier transform of distributions to the study of functions analytic in the interior of a strip in the complex plane, and having a very general type of boundary value on the sides of the strip. We show that the functions considered are uniquely determined by their boundary values. We also establish a generalization of Cauchy's formula, by means of which we establish a generalization of the following theorem of Phragmen and Lindelöf: Let $F(z)$ be analytic in the

Received by the editors September 21,1955; the original manuscript of this paper was lost and this caused considerable delay in publication.

(1) Work partially supported by National Science Foundation Grant NSF5-G1010. 
strip $a<g(z)<b$, continuous in the closed strip and satisfy $|F(x+a i)| \leqq M$, $|F(x+b i)| \leqq M$ for all real $x$. Then, if $F$ is not "too large" at infinity in the strip $a \leqq \mathscr{I}(z) \leqq b$, we have $|F(z)| \leqq M$ whenever $z$ lies in the above strip (see [8]). This theorem states that if the restriction of $F(z)$ to the two lines $\mathscr{g}(z)=a, \mathfrak{g}(z)=b$ lies in the space $L_{\infty}$, then the restriction of $F(z)$ to any line $\mathfrak{g}(z)=c$, where $a \leqq c \leqq b$, is also in $L_{\infty}$. We show that the space $L_{\infty}$ may be replaced by any of a very general class of spaces, e.g. the spaces $L_{p}$ for $1 \leqq p \leqq \infty$, or the Fourier transform of these spaces. Our method of proof is of interest since it is very different from the classical proofs.

The notation in this paper will be that of Part I (see [5]). In particular, $R(C)$ is real (complex) Euclidean space of dimension $n$. For each $l<0, D_{l}$ is space of indefinitely differentiable functions $f$ on $R$ whose carriers are contained in the cube, center origin, side $2 l ; D$ is the inductive limit of the space $D_{l}$ for $l$ integral. For each $l, D_{l}$ is the Fourier transform of the space $D_{l}$, so $D_{l}$ consists of all entire functions of exponential type $\leqq l$ which are rapidly decreasing on $R ; D$ is the inductive limit of the spaces $D_{l}$ for $l$ integral and so is the Fourier transform of $D$.

Theorems, Propositions, etc. will be numbered consecutively in each section, and all cross references will refer to the section in which they are made unless the contrary is specifically stated.

2. Analytic vector-valued maps. We shall develop the theory of analytic maps of $C$ (or a subregion) in to a complete, locally convex, topological vector space. Following a suggestion of Professor Chevalley, we shall use Morera's theorem as the defining property of analytic maps, because, as will be seen later, it is usually the simplest property to verify. Naturally, no claim is made to originality of the results of the first part of this section, although we expect the later introduced concepts of an analytic function on an arbitrary topological vector space and of an analytic map of one topological vector space into another to play an important role in the further development of the theory of distributions, especially in regard to nonlinear partial differential equations.

For simplicity, in this section we shall restrict ourselves to the case $n=1$, although our results can be extended to arbitrary $n$ and even to more general situations.

By "curve" we shall mean a simple rectifiable Jordan curve. When we speak of a closed curve in a region $A$, we shall assume that it is contractible to a point in $A$ and that its orientation is positive. The terminology in what follows is that of [1].

If $\Gamma$ is a curve, then the map $f \rightarrow \int_{\Gamma} f(z) d z$ for $f$ a continuous function on $C$ is clearly a measure of compact carrier on $C$ which we denote by $p_{\Gamma}$.

Let $V$ be a complete, locally convex, topological vector space, $A$ a region in $C$, and $t$ a map of $A$ into $V$. We shall say that $t$ is an analytic map of $A$ into $V$ if 
1. $t$ is integrable with respect to $p_{\Gamma}$ whenever $\Gamma \subset A$.

2. The image under $t$ of every compact subset of $A$ is bounded in $V$.

3. If $\Gamma$ is closed, $\int t d p=0$.

Note that Conditions 1 and 2 are always satisfied if $t$ is continuous. We call an analytic map $t: C \rightarrow V$ an entire map.

If $t: A \rightarrow V$, we shall of ten write $\int_{\mathrm{r}} t(z) d z$ for $\int t d p$.

Proposition 1. Let $t$ be a map of $A$ into $V$ which satisfies Conditions 1, 2 above. Then $t$ is analytic if and only if

$3^{\prime}$. For every $v^{\prime} \in V^{\prime}, v^{\prime} t$ is an analytic function on $A$.

Proof. Suppose first that $t$ is analytic. Then for any $v^{\prime} \in V^{\prime}$ and $\Gamma$ any closed curve in $A$, by [1],

$$
\int v^{\prime} t d p=v^{\prime} \int t d p=0 .
$$

It follows from Morera's theorem that $v^{\prime} t$ is an analytic function in $A$.

The converse follows immediately from the definition of the integral (see [1]).

Proposition 2 (Cauchy's formula). Let $t: A \rightarrow V$ be analytic. Then for any $z \in A$,

$$
t(z)=\frac{1}{i} \int_{\mathrm{r}} \frac{t(w)}{w-z} d w
$$

where $\Gamma$ is any closed curve containing the point $z$ in its interior.

Note the absence of the usual factor $2 \pi$ because of our choice of measure (see $\$ 2$ of [5]).

Proof. For any $v^{\prime} \in V^{\prime}$,

$$
v^{\prime} \cdot\left(\frac{1}{i} \int_{\Gamma} \frac{t(w)}{w-z} d w\right)=\frac{1}{i} \int_{\Gamma} \frac{v^{\prime} \cdot t(w)}{w-z} d w=\left(v^{\prime} t\right)(z)
$$

from which the result follows.

Proposition 3. Let $t: A \rightarrow V$ be analytic. Then for any $x \in A$,

$$
\lim _{h \rightarrow 0, x+h \in A} \frac{t(x+h)-t(x)}{h}=t^{(1)}(x)
$$

exists and defines an analytic map $t^{(1)}: A \rightarrow V$ (which we call the derivative of $t$ ). Thus, $t$ has derivatives of all orders $t^{(k)}$ which are analytic maps of $A \rightarrow V\left(t^{(k)}\right.$ is defined, for $k>0$ as the derivative of $t^{(k-1)}$, with $\left.t^{(0)}=t\right)$. For each $k, x \in A$,

$$
t^{(k)}(x)=\frac{k !}{i} \int_{\Gamma} \frac{t(w)}{(w-x)^{k+1}} d w
$$


where $\Gamma$ is any closed curve containing $x$ in its interior.

Proof. Let $x \in A, x+h \in A$. By Proposition 2,

$$
\begin{aligned}
\frac{t(x+h)-t(x)}{h} & =\frac{1}{i h} \int_{\Gamma} \frac{t(w)}{(w-x-h)} d w-\frac{1}{i h} \int_{\Gamma} \frac{t(w)}{w-x} d w \\
& =\frac{1}{i} \int_{\Gamma} \frac{t(w)}{(w-x)(w-x-h)} d w
\end{aligned}
$$

where $\Gamma$ is any closed curve containing $x$ and $x+h$ in its interior. (We assume that $|h|$ is so small that, for all $\left|h^{\prime}\right| \leqq|h|, x+h^{\prime}$ lies in the interior of $\Gamma$.) It follows immediately that $\int_{\Gamma}(t(w) /(w-x)(w-x-h)) d w$ depends continuously on $h$, for such $h$ as we are considering. Hence,

$$
\lim _{h \rightarrow 0, x+h \in A} \frac{t(x+h)-t(x)}{h}=\frac{1}{i} \int_{\Gamma} \frac{t(w)}{(w-x)^{2}} d w .
$$

A simple induction concludes the proof of the proposition.

Proposition 4. Let $t: A \rightarrow V$. A necessary and sufficient condition that $t$ be analytic is that, for each $x \in A$, the limit

$$
\lim _{h \rightarrow 0, x+h \in A} \frac{t(x+h)-t(x)}{h}
$$

exists.

Proof. The necessity was demonstrated in Proposition 3, so we demonstrate the sufficiency. If the limit exists, then we know that $t$ is continuous at each $x \in A$. Further, since the limit exists, we know that, for each $v^{\prime} \in V^{\prime}$, $x \in A$, the $\operatorname{limit} \lim _{h \rightarrow 0, x+h \in A}\left(v^{\prime} t(x+h)-v^{\prime} t(x)\right) / h$ exists. Thus, $v^{\prime} t$ is an analytic function on $A$. Combining this with Proposition 2, we obtain our result.

COROLlary. Every analytic map is continuous.

Proposition 5 (TAylor's Formula). Let $t: A \rightarrow V$ be analytic and let $a \in A$; call $\rho$ the distance of $a$ to the complement of $A$. Then for all $|z-a|<\rho$,

$$
t(z)=t(a)+(z-a) t^{(1)}(a)+\cdots+\frac{(z-a)^{k}}{k !} t^{(k)}(a)+\cdots
$$

where the series converges uniformly in $|z-a| \leqq \rho^{\prime}<\rho$.

Proof. Let $\rho^{\prime}<\rho$; the series

$$
\frac{1}{w-a}+\frac{z-a}{(w-a)^{2}}+\cdots+\frac{(z-a)^{k}}{(w-a)^{k+1}}+\cdots
$$

converges uniformly to $1 /(w-z)$ for $w$ on the circle $|w-a|=\rho^{\prime}+\left(\rho-\rho^{\prime}\right) / 2$ 
and $|z-a| \leqq \rho^{\prime}$. Let $W$ be the space of functions analytic in the circle $|w-a|$ $<\rho^{\prime}+\left(\rho-\rho^{\prime}\right) / 2$ and continuous on the boundary, with the topology of uniform convergence on the circle $\Gamma:|w-a|=\rho^{\prime}+\left(\rho-\rho^{\prime}\right) / 2$ so $W$ is a Banach space. Moreover, for each $z$ with $|z-a| \leqq \rho^{\prime}$, the series (1) converges in $W$, and uniformly so for $|z-a| \leqq \rho^{\prime}$. Now, $f \rightarrow i^{-1} \int_{\mathrm{r}} t(w) f(w) d w$ is a continuous linear map of $W \rightarrow V$. Thus, for each $z$ with $|z-a| \leqq \rho^{\prime}$,

$$
\begin{aligned}
\frac{1}{i} \int_{\Gamma} \frac{t(w)}{w-z} d w= & \frac{1}{i} \int_{\Gamma} \frac{t(w)}{w-a} d w+\frac{(z-a)}{i} \int_{\Gamma} \frac{t(w)}{(w-a)^{2}} d w+\cdots \\
& +\frac{(z-a)^{k}}{i} \int_{\Gamma} \frac{t(w)}{(w-a)^{k+1}} d w+\cdots
\end{aligned}
$$

where the series converges uniformly in $|z-a| \leqq \rho^{\prime}$. The result now follows from Proposition 3.

Proposition 6. Let $U$ be a filter of analytic maps $t_{b}: A \rightarrow V$. Suppose $U$ converges to a map $t: A \rightarrow V$ uniformly on every compact set of $A$. Then $t$ is an analytic map.

Proof. By the corollary to Proposition 4, each $t_{b}$ is continuous, so that $t$ is continuous. For any $v^{\prime} \in V^{\prime}$, denote by $v^{\prime} U$ the filter base obtained from $U$ by replacing each $t_{b}$ of $U$ by $v^{\prime} t_{b}$. Then $v^{\prime} U$ converges to $v^{\prime} t$ uniformly on every compact set of $A$, so that $v^{\prime} t$ is analytic. Combining the above with Proposition 1 , we have our result. .

Proposition 7 (Liouville's THEOREM). Let $t$ be an entire map into $V$ such that $\{t(z)\}_{\boldsymbol{z} \in C}$ is bounded in $V$, or even weakly bounded. Then $t$ is a constant.

Proof. Assume there exist $z_{1}, z_{2} \in C$ such that $t\left(z_{1}\right) \neq t\left(z_{2}\right)$. Then for some $v^{\prime} \in V^{\prime},\left(v^{\prime} t\right)\left(z_{1}\right) \neq\left(v^{\prime} t\right)\left(z_{2}\right)$. But $v^{\prime} t$ is an entire bounded function, hence it is a constant. This contradiction proves our result.

Lemma 1. Let $A$ be a region bounded by a closed curve $\Gamma$. Let $t$ be a continuous map of $\bar{A}$ (the closure of $A$ ) into $V$ whose restriction to $A$ is analytic. Then, for any $z \in A$,

$$
t(z)=\frac{1}{i} \int_{\mathrm{r}} \frac{t(w)}{w-z} d w .
$$

Proof. For any $v^{\prime} \in V^{\prime}$,

$$
v^{\prime} \cdot\left(\frac{1}{i} \int_{\Gamma} \frac{t(w)}{w-z} d w\right)=\frac{1}{i} \int_{\Gamma} \frac{v^{\prime} t(w)}{w-z} d w=v^{\prime} \cdot t(z)
$$

from which the result follows.

Proposition 8. Let $B$ be a topological space, and let $A$ be a region bounded by a closed curve $\Gamma$. Let $t$ be a map of $\bar{A} \times B$ into $V$ such that the restriction of 
$t$ to $\Gamma \times B$ is continuous. For each $b \in B$, denote by $t_{b}$ the map of $\bar{A} \rightarrow V: t_{b}(a)$ $=t(a, b)$; suppose that each $t_{b}$ is continuous and that its restriction to $A$ is analytic. Then the restriction of $t$ to $A \times B$ is continuous.

Proof. For each $a \in A, b \in B$ we may write, by Lemma $1, t_{b}(a)$ $=i^{-1} \int_{\Gamma}\left(t_{b}(a) /(z-a)\right) d z$. It follows that $b \rightarrow t_{b}(x)$ are equicontinuous maps of $B$ into $V$ for $x$ in any compact set of $A$. This implies the truth of Proposition 8 .

Proposition 9 (Maximum modulus principle). Let $A$ be the region bounded by a curve $\Gamma$. Let $t$ be a continuous map of $\bar{A}$ into $V$ whose restriction to $A$ is analytic. Then $\{t(z)\}_{z \in \bar{A}}$ is contained in the smallest closed, convex, circled set containing $\{t(z)\}_{z \in \mathrm{r}}$.

Proof. What we must show is the following: Let $v^{\prime} \in V^{\prime}$ and $a>0$ be so chosen that $\left|v^{\prime} \cdot t(z)\right| \leqq a$ for all $z \in \Gamma$; then also $\left|v^{\prime} \cdot t(z)\right| \leqq a$ for all $z \in \bar{A}$. But, $v^{\prime} t$ is an analytic function on $A$ which is continuous on $\bar{A}$; the result follows from the classical maximum modulus theorem.

Corollary. Let $A$ be the region bounded by a curve $\Gamma$; let $B$ be a topological space, and let $t: \bar{A} \times B \rightarrow V$. Suppose that, for some $b_{0} \in B, v_{0} \in V, \lim _{b \rightarrow b_{0}} t(x, b)$ $=v_{0}$ uniformly for $x \in \Gamma$. Then also $\lim _{b \rightarrow b_{0}} t(x, b)=v_{0}$ uniformly for $x \in \bar{A}$.

Proof. By a preliminary transformation, we may restrict ourselves to the case $v_{0}=0$; the result is an immediate consequence of Proposition 9.

Let us denote by $a_{A V}$ the space of all analytic maps of $A$ into $V$. Let $W$ be another complete, locally convex, topological vector space, and let $s: V \rightarrow W$. We shall say that $s$ is an analytic map over $A$ if, for any $t \in a_{A V}$, $s t \in a_{A W}$.

Proposition 10. For any $A$ and any $V, s$ is an analytic map over $A$ of $C$ into $V$ if and only if $s$ is an entire map.

Proof. Suppose first that $s$ is an entire map. Then for any $t \in Q_{A C}$, st is a continuous map of $A$ into $V$. For any $v^{\prime} \in V^{\prime}$, we know that $v^{\prime}$ s is an entire function. Thus, $v^{\prime} s t$ is an analytic function on $A$; this means that $s t \in Q_{A V}$, i.e. $s$ is an analytic map over $A$.

Conversely, if $s$ is analytic over $A$, then for any $v^{\prime} \in V^{\prime}, v^{\prime} s$ is a function on $C$ with the property that $w^{\prime} s t$ is an analytic function on $A$ whenever $t$ is an analytic function on $A$. This clearly implies that $w^{\prime} s$ is an entire function; since $s$ is continuous, the result follows from Proposition 1.

The next two propositions are easily verified.

Proposition 11. For any $v_{0} \in V$, and any region $A$, the map $v \rightarrow v+v_{0}$ is analytic over $A$.

Proposition 12. For any $A, V, W$, let $s: V \rightarrow W$ be a continuous linear map. Then $s$ is analytic over $A$. 
Proposition 13 (Liouville's THEOREM). Let $t: V \rightarrow W$ be an analytic map over $C$ such that the image of $V$ under $t$ is weakly bounded. Then $t$ is a constant.

Proof. Assume $t$ is not a constant and let $v_{1}, v_{2} \in V$ be so chosen that $t\left(v_{1}\right) \neq t\left(v_{2}\right)$. Let $s: C \rightarrow V$ be the entire map $s(z)=z v_{1}+(1-z) v_{2}$. Then $t s$ is an entire nonconstant map into $W$ whose image is weakly bounded. This contradicts Proposition 7 and so concludes the proof.

Suppose $W$ is a topological ring, and for $w_{1}, w_{2} \in W$ let $w_{1} \circ w_{2}$ be their product. Then for any region $A$ the space $a_{A W}$ can be made into a ring by defining the product $t_{1} \circ t_{2}$ of any $t_{1}, t_{2} \in Q_{A W}$ to be the map $z \rightarrow t_{1}(z) \circ t_{2}(z)$. It follows easily from Proposition 4 that $t_{1} \circ t_{2}$ is analytic, and that its derivative is just $t_{1} \circ t_{2}^{(1)}+t_{1}^{(1)} \circ t_{2}$. Again, for any $V$, the space of analytic maps of $V$ into $W$ over $A$ can be made, naturally into a ring. In particular, if $W=C$, we obtain the ring of analytic functions on $V$. We can put various natural topologies on these rings and obtain, in this way, interesting types of topological rings.

Instead of considering analytic maps of $A \rightarrow V$ we can consider certain classes of entire mappings of $C \rightarrow V$ defined by certain properties of the coefficients of their Taylor expansions (e.g. an analog of the class of entire functions of exponential type). We can consider similar notions in discussing maps of $V \rightarrow W$ (e.g. maps $s: V \rightarrow W$ such that $s t$ is an entire mapping into $W$ for every continuous linear map $t: C \rightarrow V$.) In any case, the main sought-after property is the analog of the Taylor expansion for certain types of analytic maps of $V \rightarrow W$. The problem is of the following form: Suppose $W$ is a topological ring. When is every analytic map of $V$ into $W$ (of a suitable type) a limit (in some sense) of linear combinations of constants and products of continuous linear maps, i.e. when is the ring generated by constants and continuous linear maps dense in the ring of all analytic maps (of a suitable class)? The problem can be answered, at least partially, in case that $W=C$ and $V$ is nuclear, e.g. $V=\mathbb{D}$.

The method of deriving such results is the following: Let $f$ be an analytic function on $\mathbb{D}$ over $C$. Then we define for any $n$ and any $v \in \mathbb{D},\left(\partial^{n} f\right)(v ;)$ as an $n$-linear function on $D$ by: $\left(\partial^{0} f\right)(v ; a)=f(v)$;

$$
\begin{aligned}
\left(\partial^{n} f\right)\left(v ; a_{1}, \cdots, a_{n}\right) & \\
& =\lim _{h \rightarrow 0} \frac{\left(\partial^{n-1} f\right)\left(v+h a_{n} ; a_{1}, \cdots, a_{n-1}\right)-\left(\partial^{n-1} f\right)\left(v ; a_{1}, \cdots, a_{n-1}\right)}{h}
\end{aligned}
$$

for any $a, a_{1}, \cdots, a_{n} \in \mathcal{D} .\left(\partial^{n} f\right)(v ;)$ is an $n$-linear function and $\partial^{n} f$ is analytic on $D \times D \times \cdots \times D(n+1$ factors $)$ over $C$. By considering the linear maps $\lambda \rightarrow \lambda a$ of $C \rightarrow D$ we can prove easily that $\sum\left(\partial^{n} f\right)(0 ; a, a, \cdots, a) / n$ ! converges to $f(a)$ uniformly for $a$ in any bounded set of $D$.

The Schwartz kernel theorem (see Espaces de fonctions différentiables à valeurs vectorielles, J. Analyse Math. vol. 4 (1954-1955) pp. 87-148) tells us that each $\left(\partial^{n} f\right)(0 ;)$ is a distribution on real Euclidean space of $n$ dimen- 
sions. Using Fourier series, it is not hard to prove that the restriction of the distribution $\left(\partial^{n} f\right)(0 ;)$ to $\mathbb{D} \times \cdots \times \mathbb{D}(n$ factors $)$ is the limit of polynomials on $D$ of degree $n$. (Compare an analogous argument in my paper, On the theory of kernels of Schwartz, Proc. Amer. Math. Soc. vol. 7 (1956) pp. 713718). From this we derive the fact that $f$ is the limit of polynomials.

In case that $V$ is a complex Euclidean space, then, instead of considering all analytic maps of $A$ into $V$, we can consider analytic maps of $A$ into a subset $U$ of $V$, i.e. analytic maps $t: A \rightarrow V$ for which $t(A) \subset U$. Let $W$ be a complete topological vector space; we call a map $s: U \rightarrow W$ such that, if $t$ is an analytic map of $A$ into $U$ then it is an analytic map of $A$ into $W$, an analytic map of $U$ into $W$, (over $A$ ). It can be shown that the set of analytic maps of $U \rightarrow W$ over $A$ is the same for all $A$ which are relatively compact, we call these simply the analytic maps of $U \rightarrow W$. We can again introduce natural topologies and obtain interesting types of topological vector spaces and rings. We hope to discuss all these topics in great detail at a later date.

Theorem 1. Let $F \in D$; then $z \rightarrow \tau_{z} F$ is an entire map into D. Similar results hold for the spaces $D^{\prime}, E, E^{\prime}$ in place of $D$.

(For $S \in D^{\prime}, z \in C$, we define $\tau_{z} S \in D^{\prime}$ by $\tau_{z} S \cdot G=S \cdot \tau_{z} G$.)

Proof. (a) We know that $z \rightarrow \exp (i z \cdot) f$ is a continuous map of $C$ into $D$. Thus,

$$
z \rightarrow \mathcal{F}[\exp (i z \cdot) f]=\tau_{z} F
$$

is a continuous map of $C$ into $D$. If $\Gamma$ is any closed curve in $C$, then for any $x \in C$

$$
\begin{aligned}
\left(\int_{\mathrm{\Gamma}} \tau_{z} F d z\right)(x) d z & =\int_{\Gamma}\left(\tau_{z} F\right)(x) d z \\
& =\int_{\mathrm{r}} F(x-z) d z \\
& =0
\end{aligned}
$$

because $F$ is an entire function. Thus, $z \rightarrow \tau_{z} F$ is entire.

(b) If $S \in D^{\prime}$, the continuity of $z \rightarrow \tau_{z} S$ follows from the continuity of $z \rightarrow \exp (-i \bar{z}) T$ (for $T \in D^{\prime}$ ) of $C$ into $\mathscr{D}^{\prime}$, and the continuity of $T \rightarrow F(T)$ of $D^{\prime}$ into $D^{\prime}$ (see [5, Proposition 1 of $\S 3$ ]. For $\Gamma$ any closed curve in $C$ and any $G \in D$,

$$
\begin{aligned}
\left(\int_{\Gamma} \tau_{z} S d z\right) \cdot G & =\int_{\Gamma} \tau_{z} S \cdot G d z=\int_{\Gamma} S \cdot \tau_{-z} G d z \\
& =S \cdot \int_{\Gamma} \tau_{-2} G d z=0
\end{aligned}
$$


by part (a) above. Thus, $z \rightarrow \tau_{z} S$ is entire.

(c) The proofs for the spaces $E, E^{\prime}$ are similar.

Theorem 2. For any $F \in D$, let $t: C \rightarrow D$ be the map $t(z)=\tau_{s} F$. Then, for any $k \geqq 0, t^{(k)}(z)=\tau_{z} F^{(k)}$. If $S \in D^{\prime}$, and if $s: C \rightarrow D^{\prime}$ is the map $s(z)=\tau_{z} S$, then $s^{(k)}(z)=\tau_{z} S^{(k)}$ where $S^{(k)} \cdot G=(-1)^{k} S \cdot G^{(k)}$ for any $G \in D$. Similar results hold for the spaces $E, E^{\prime}$. on $D$,

Proof. (a) For any $x, z \in C$, since $H \rightarrow H(x)$ is a continuous linear function

$$
\begin{aligned}
\lim _{h \rightarrow 0} \frac{\tau_{z+h} F-\tau_{z} F}{h}(x) & =\lim _{h \rightarrow 0} \frac{F(x-z-h)-F(x-z)}{h} \\
& =F^{(1)}(x-z) \\
& =\left(\tau_{z} F^{(1)}\right)(x) .
\end{aligned}
$$

Thus, $t^{(1)}(z)=\tau_{z} F^{(1)}$. The case $k>1$ is proven by induction. $D^{\prime}$,

(b) For any $z \in C, G \in D$, since $S \rightarrow S \cdot G$ is a continuous linear function on

$$
\begin{aligned}
\lim _{h \rightarrow 0} \frac{\tau_{z+h} S-\tau_{z} S}{h} \cdot G & =\lim _{h \rightarrow 0} \frac{\tau_{z+h} S \cdot G-\tau_{z} S \cdot G}{h} \\
& =\lim _{h \rightarrow 0} \frac{S \cdot \tau_{-z-h} G-S \cdot \tau_{-z} G}{h} \\
& =S \cdot \lim _{h \rightarrow 0} \tau_{-z-h} G-\tau_{-z} G \\
& =-S \cdot \tau_{-z} G^{(1)} \\
& =-S \cdot\left(\tau_{-z} G\right)^{(1)}
\end{aligned}
$$

by the result of part (a) above. Thus, $\left(s^{(1)}\right)(z)=-\tau_{z} S^{(1)}$. The proof for $k>1$ is an easy induction.

(c) The proofs for the spaces $E, E^{\prime}$ are similar.

Corollary. For any $F \in D$, the series $\sum z^{k} F^{(k)} / k$ ! converges to $\tau_{z} F$ uniformly for $z$ in any compact set of $C$. Similar results hold for the spaces $D^{\prime}, E, E^{\prime}$.

Let $S \in D^{\prime}$ be such that $\left\{\tau_{z} S\right\}_{z \in C}$ is weakly bounded in $D^{\prime}$. Then Proposition 7 and Theorem 1 show us that $\tau_{s} S=S$ for all $z \in C$. We claim that $S=a \mathfrak{q}(1)$ where $a$ is a constant. Let $T$ be the inverse Fourier transform of $S$; we show first that the carrier of $T$ (see [6]) is just the point 0 . Let $f \in D$ vanish for all $x \leqq \delta>0$. Then clearly the sequence $g_{n}=\exp (-n \cdot) f$ converges to zero in D. Thus, $G_{n}=\tau_{i n} F \rightarrow 0$ in $D$, so that $S \cdot G_{n} \rightarrow 0$. But,

$$
S \cdot G_{n}=\tau_{-i n} S \cdot F=S \cdot F .
$$

Thus, $S \cdot F=0$, which means that the carrier of $T$ is contained in the half-line 
$[-\infty<x \leqq 0]$. A similar calculation shows that the carrier of $T$ must be contained in the half-line $[0 \leqq x<\infty]$; thus, the carrier of $T$ is just the point 0 . Thus, we may write (see [6]) $T=\sum_{j=0}^{r} a_{j} \delta^{(j)}$ where $a_{j}$ are complex numbers and $\delta^{(j)}$ is the $j$ th derivative of the Dirac measure. Let $f \in D$ be 1 on a neighborhood of the origin. Then, for any $y \in R$,

Since

$$
\exp (y \cdot) T \cdot f=\sum a_{j} \delta^{(j)} \cdot \exp (y \cdot)=\sum a_{j} y^{i} .
$$

$$
F[\exp (y \cdot) T]=\tau_{-i y} S=S,
$$

it follows that $\sum a_{j} y^{j}$ is constant for all real $y$. This clearly implies $a_{j}=0$ for $>0$. Thus, $T=a_{0} \delta$, or $S=a_{0} q(1)$. We have proven:

Theorem 3. Suppose for some $S \in D^{\prime},\left\{\tau_{z} S\right\}_{z \in C}$ is weakly bounded in $D^{\prime}$. Then $S$ is a constant, i.e. $S=a q(1)$ for some $a \in C$.

Corollary. Let $S \in D^{\prime}$ be doubly periodic, that is, suppose there are $a_{1}$, $a_{2} \in C$ which are linearly independent over $R$ and such that $\tau_{a_{1}} S=\tau_{a_{2}} S=S$. Then $S$ is a constant.

Proof. Let $P$ be the "period parallelogram" for $S$, i.e. $P$ is the closed parallelogram in $C$ with vertices $0, a_{1}, a_{2}, a_{1}+a_{2}$. Then for any $z \in C$ there is a $y \in P$ such that $\tau_{z} S=\tau_{y} S$. Thus, the set $\left\{\tau_{z} S\right\}_{z \in C}$ is the same as $\left\{\tau_{y} S\right\}_{y \in P \text {. }}$ But, the latter is compact, hence bounded, in $D^{\prime}$ because of the continuity of $z \rightarrow \tau_{z} S$ (see the proof of Theorem 1). Thus, in virtue of Theorem $3, S$ is a constant.

THEOREM 4. Let $S \in D^{\prime}$ have the property that, for some sequence of positive numbers $a_{k} \rightarrow \infty,\left\{\tau_{-i a_{k}} S\right\}$ is bounded in $D^{\prime}$. Then, if $T$ denotes the inverse Fourier transform of $S$, the carrier of $T$ is contained in the half-axis $[0 \leqq x<\infty]$. Conversely, if the carrier of $T$ is contained in $[0<x<\infty]$, then for any real number $b\left\{\tau_{-i a} S\right\}_{a \geq b}$ is bounded in $D^{\prime}$.

Proof. We demonstrate the converse first. If the carrier of $T$ is contained in $[0<x<\infty]$ then, since the carrier of a distribution is a closed set, there is a $\rho>0$ so that the carrier of $T$ is contained in $[\rho \leqq x<\infty]$. From this it follows easily that $\{\exp (-a \cdot) T\}_{a \geq b}$ is bounded in $D^{\prime}$. Thus, $\left\{\tau_{-i a} S\right\}_{a \geq b}$ is bounded in $D^{\prime}$.

On the other hand, suppose $\left\{\tau_{-i a_{k}} S\right\}$ is bounded in $D^{\prime}$. For any $f \in \mathscr{D}$ which vanishes for $x \geqq \rho<0$, it is clear that $\exp \left(a_{k} \cdot\right) f \rightarrow 0$ in $D$. Thus,

$$
S \cdot \mathcal{F}\left(\exp \left(a_{k} \cdot\right) f\right)=S \cdot \tau_{-i a_{k}} F \rightarrow 0 .
$$

Moreover, we even have $\tau_{-i a \mathfrak{l}} S \cdot \tau_{-i a_{k}} F \rightarrow 0$ uniformly in $\mathfrak{l}$ as $k \rightarrow \infty$. In particular, $\tau_{-i a_{k}} S \cdot \tau_{-i a_{k}} F \rightarrow 0$. But,

$$
\begin{aligned}
\tau_{-i a_{k}} S \cdot \tau_{-i a_{k}} F & =S \cdot \tau_{i a_{k}}\left(\tau_{-i a_{k}} F\right) \\
& =S \cdot F .
\end{aligned}
$$


Thus, $S \cdot F=0$ which means that the carrier of $T$ is contained in $[0 \leqq x<\infty]$.

Let $h$ be an entire function on $C$. For any $f \in D$, we define $f^{h} \in D$ as follows: Let $h(z)=\sum a_{j} z^{i}$ be the Taylor expansion of $h$. Then we define $f^{h}$ $=\sum a_{j} f^{j}$; we must show that this series converges in $D$. To this end, we note that if $f \in D_{\mathfrak{l}}$ then all $f^{j} \in D_{\mathfrak{l}}$. Further, for any $k, j$

$$
\begin{aligned}
& \max _{x \in R}\left|\left(\frac{d^{k}}{d X^{k}} f^{j}\right)(x)\right| \\
& \quad \leqq a \max _{x \in R,-1 k_{1} \varliminf_{k}, \cdots,-1 \varliminf_{j} \leqq_{k}}\left|\left(\frac{d^{k_{1}}}{d X^{k_{1}}} f\right)(x)\right|\left|\left(\frac{d^{k_{2}}}{d X^{k_{2}}} f\right)(x)\right|^{2}\left|\left(\frac{d^{k_{j}}}{d X^{k_{j}}} f\right)(x)\right|^{\prime}
\end{aligned}
$$

where $a$ is a positive number depending on $k$ but not on $j$ or $f$, and where the -1 st derivative of $f$ is defined to be 1 . Thus,

$$
\max _{x \in R}\left|\left(\frac{d^{k}}{d X^{k}} f^{i}\right)(x)\right| \leqq b^{j}
$$

where $b$ is a positive number which depends on $k$ and the first $k$ derivatives of $f$. Moreover, if $B$ is any bounded set in $D$, it is clear that we can choose a $b>0$ so that

$$
\max _{x \in R}\left|\left(\frac{d^{k}}{d X^{k}} f^{i}\right)(x)\right| \leqq b^{i}
$$

for all $f \in B$. Thus, the series $\sum a_{j} f^{j}$ converge in $D$ uniformly for $f$ in any bounded set of $D$.

Let $\left\{g_{p}\right\}$ be a sequence of functions of $D_{\mathfrak{l}}$ such that $g_{p} \rightarrow g$ in $D_{\mathfrak{l}}$. Let $N$ be a convex neighborhood of zero in $D_{\mathfrak{l}}$. Since the set of functions $\left\{g_{p}\right\}$ is bounded in $D_{\mathfrak{l}}$ we can, by the above, find an integer $J$ so large that for all $p$

$$
\sum_{j \geqq J} a_{j} g_{p} j \in \frac{1}{4} N, \quad \sum_{j \geqq J} a_{j} g^{j} \in \frac{1}{4} N .
$$

Next, we can choose an integer $P$ so large that, for all $p \geqq P$,

$$
\sum_{j<J} a_{j} g_{p j} \in \sum_{j<J} a_{j} g^{j}+\frac{1}{4} N .
$$

Thus, for all $p \geqq P$,

$$
g_{p}^{h}=\sum a_{j} g_{p}^{j} \in g^{h}+N .
$$

This means that $g_{p}^{h} \rightarrow g^{h}$ in $D_{l}$. Since $D_{\mathfrak{l}}$ is metrizable $g \rightarrow g^{h}$ is a continuous map of $D_{1}$ into itself. Thus (see [2]) $g \rightarrow g^{h}$ is a continuous map of $D \rightarrow D$.

Now, let $A$ be a region in $C$ and $t: A \rightarrow D$ an analytic map. Then we claim that $z \rightarrow t(z)^{h}$ is an analytic map of $A$ into D. For, since $t$ is continuous, the 
image under $t$ of any compact set in $A$ is bounded in $\mathfrak{D}$. Thus, $\sum a_{j} t(z)^{j}$ converges uniformly for $z$ in any compact set of $A$. Further, each partial sum of $\sum a_{j} t(z)^{j}$ is an analytic map of $A \rightarrow D$ since $D$ is a topological ring. Thus, by Proposition $6, z \rightarrow t(z)^{h}$ is an analytic map of $A \rightarrow D$.

We have proven

TheOREM 5. $f \rightarrow f^{h}$ is an analytic map of $D$ into $D$ over any region $A$.

Since $\mathcal{F}: D \rightarrow D$ is a continuous linear map, hence an analytic map, and since the composition of two analytic maps is clearly analytic, we have

Corollary. For $F \in D$, let $F^{* h}=\sum a_{j} F^{*_{i}}$ (where $F^{*_{i}}$ is the convolution of $F$ with itself $j$ times). Then the series $\sum a_{j} F^{* i}$ converge uniformly in $D$ for $F$ in any bounded set of $D$. Moreover, $F \rightarrow F^{* h}$ is an analytic map of $D \rightarrow D$ over any region $A$.

We can perform a similar process for the spaces $E, E$ instead of $D, D$. Since every measure of compact carrier on $C$ is contained in $E$, we can thus define, for example, the exponential of such a measure. For example, if we consider the element $\delta^{(1)} \in E$ (derivative of the Dirac measure) then we find as in $\S 3$ of $[5],\left[\delta^{(1)}\right] \exp =\delta_{1}$. More generally, for any $a \in C$

$$
\left[\delta_{a}^{(1)}\right]^{\exp }=\delta_{a+1} .
$$

We restate the above as

THEOREM 6. $f \rightarrow f^{h}$ and $F \rightarrow F^{* h}$ are analytic maps over any region $A \subset C$ of $\mathcal{E} \rightarrow \mathcal{E}$ and $E \rightarrow E$ respectively. We have $F\left(f^{h}\right)=F^{* h}$.

Proposition 14. For any $a, b \in C$,

$$
\left[\delta_{a}^{(1)}\right]^{\exp (b \cdot)}=\delta_{a+b}
$$

Let $V$ be a complete, locally convex, topological vector space and let $t: C \rightarrow V$ be an entire map of $C \rightarrow V$. Suppose that, for every $v^{\prime} \in V^{\prime}, v^{\prime} t$ is an entire function of exponential type, and that there is a $b^{\prime}>0$ so that all $v^{\prime} t$ for $v^{\prime} \in V^{\prime}$ are of exponential type $\leqq b^{\prime}$. Then we say that $t$ is an entire map of exponential type $\leqq b^{\prime}$; the g.l.b. of all $b^{\prime}$ satisfying the above condition is called the exponential type of $t$.

Let $V$ be a complete, locally convex, topological vector space and $s: R \rightarrow V$ a continuous map. We say that $s$ is differentiable at $x \in R$ if

$$
\lim (s(x+h)-s(x)) / h=s^{(1)}(x)
$$

exists in $V$. We define similarly $n$-times differentiable maps and indefinitely differentiable maps $s$ of $R \rightarrow C$, and the derivatives $s^{(k)}$ of $s$.

By the carrier of a map $s: R \rightarrow V$ we mean the closure of the set of $x \in R$ such that $s(x) \neq 0$. Suppose that $V$ is metrizable. Then we define the space 
$D_{l}(V)$ as consisting of all indefinitely differentiable maps of $R \rightarrow V$ whose carriers are contained in $[-l \leqq x \leqq l]$. The topology of $D_{l}(V)$ is defined by means of the semi-norms

$$
\rho_{i j}(s)=\sup _{x \in R} \rho_{j}\left(s^{(i)}(x)\right)
$$

where $\rho_{j}$ is a sequence of semi-norms on $V$ sufficient to define the topology of $V$. It is not difficult to see that the spaces $D_{l}(V)$ are complete and that the spaces $D_{l}(V)$ for $l$ integral form a sequence of definition for a space $D(V)$ of type $(\mathscr{F})$ in the sense of Dieudonné and Schwartz (see [2]).

For any $s \in \mathscr{D}(V)$ we define its Fourier transform $\mathcal{F}(s)=t$ as the map of $C \rightarrow V: t(z)=\int s(x) e^{-i x z} d x$. We can now extend the classical Paley-Wiener theorem:

Theorem 7. Let $t$ be a map of $C \rightarrow V$. A set of necessary and sufficient conditions that $t$ be of the form $\mathcal{F}(s)$ for $s \in D_{l}(V)$ is:

(a) $t$ is an entire map of exponential type $\leqq l$.

(b) $t$ is rapidly decreasing on $R$, i.e. for any polynomial $P$ of one variable, $\{P(x) t(x)\}_{x \in R}$ is bounded in $V$.

Proof. It is easily seen that, if $t$ is of the form $\mathcal{F}(s)$ for $s \in \mathscr{D}_{l}(V)$, then condition (a) is satisfied. Condition (b) follows easily from the fact that $\mathcal{F}\left(s^{(k)}\right)(z)$ $=(i z)^{k} \mathfrak{F}(s)$ for any $k \geqq 0$.

Conversely, suppose that $t$ satisfies conditions (a) and (b). Then we verify easily (from (b)) that the map $u: R \rightarrow V$ defined by $u(y)=\int t(x) e^{i x y} d x$ is continuous. (The existence of the integral follows easily from (b).) Moreover, the integrals $\int(i x)^{k} t(x) e^{i x y} d y$ exist for each $k>0, y \in R ; u$ is indefinitely differentiable and $u^{(k)}(y)=\int(i x)^{k} t(x) e^{i x y} d x$. Furthermore, for any $v^{\prime} \in V^{\prime}, v^{\prime} t$ is, by (a), an entire function of exponential type $\leqq l$. Thus, by the classical PaleyWiener theorem (see [5]),

$$
v^{\prime}(u(y))=\int\left(v^{\prime} t\right)(x) e^{i x y} d y=0 \text { for } y \geqq l .
$$

Since this holds for all $v^{\prime} \in V^{\prime}$, we must have $u(y)=0$ for all $y \geqq l$.

It remains to show that $t=\mathcal{F}(u)$. For any $v^{\prime} \in V^{\prime}, z \in C$, by the classical Fourier inversion formula, we have

$$
v^{\prime} \int u(y) e^{-i z y} d y=\int v^{\prime}(u(y)) e^{-i z y} d y=v^{\prime}(t(z))
$$

because $v^{\prime} u(y)=\int v^{\prime} t(x) e^{i x y} d x$. Thus, by the Hahn-Banach theorem, $t(z)$ $=\int u(y) e^{-i z y} d y=(\mathcal{F}(u))(z)$ which completes the proof.

Let us denote by $D_{l}(V)$ the space of all entire maps of $C$ into $V$ of exponential type $\leqq l$ which are rapidly decreasing on $R$. The topology of $D_{l}(V)$ is defined so that $\mathcal{F}: D_{l}(V) \rightarrow D_{l}(V)$ is a topological isomorphism. Thus, $D_{l}(V)$ is a complete, metrizable, topological vector space. 
Theorem 8. Let $\left\{t_{j}\right\}$ be a sequence in $D_{l}(V)$. A necessary and sufficient condition that $t_{j} \rightarrow 0$ in $D_{l}(V)$ is: For any polynomial $P$ in one variable, $P(x) t_{j}(x)$ $\rightarrow 0$ in $V$ uniformly for $x \in R$.

Proof. For each $j$, call $s_{j}=\mathcal{F}^{-1}\left(t_{j}\right)$, so $s_{j} \in D_{l}(V)$; let $\alpha$ be a continuous seminorm on $V$. Suppose first that $s_{j} \rightarrow 0$ in $D_{l}(V)$. Then, by the definition of the topology of $D_{l}(V)$, it follows that, for any $k, s_{j}^{(k)}(x) \rightarrow 0$ in $V$ uniformly for $x \in R$. Now, it is easily seen that, for any $y \in R,\left(\mathcal{F}\left(s_{j}^{(k)}\right)\right)(y)=(i y)^{k} t_{j}(y)$. Thus, by well-known properties of the integral,

$$
\begin{aligned}
\alpha\left((i y){ }^{k} t_{j}(y)\right) & =\alpha \int s_{j}^{(k)}(x) e^{-i x y} d y \\
& \leqq \sup _{x \in R} \alpha\left(s_{j}^{(k)}(x)\right) \int_{-l}^{l} d y
\end{aligned}
$$

for any $y \in R$. It follows that, for any polynomial $P$ in one variable, $P(y) t_{j}(y)$ $\rightarrow 0$ in $V$ uniformly for $y \in R$.

Conversely, suppose that, for any polynomial $P$ in one variable, $P(y) t_{j}(y)$ $\rightarrow 0$ in $V$ uniformly for $y \in R$. Then, for any $k,(i y)^{k} t_{j}(y) \rightarrow 0$ in $V$ uniformly for $y \in R$. Thus, for any $x \in R$, as in the proof of Theorem 7 , we have $s_{j}^{(k)}(x)$ $=\int(i y)^{k} t_{j}(y) d y$. Thus,

$$
\alpha\left(s_{j}^{(k)}(x)\right)=\int y^{k} \alpha\left(t_{j}(y)\right) d y .
$$

Now, $\alpha\left(t_{j}(y)\right)$ and $\alpha\left((i y)^{k+2} t_{j}(y)\right)=y^{k+2} \alpha\left(t_{j}(y)\right) \rightarrow 0$ uniformly for $y \in R$. It follows that $\int y^{k} \alpha\left(t_{j}(y)\right) d y \rightarrow 0$ from which the result follows.

Denote by $D(V)$ the inductive limit of the spaces $D_{l}(V)$ for $l$ integral, so $D(V)$ consists of all entire maps of $C \rightarrow V$ of exponential type which are rapidly decreasing on $R$. $\mathcal{F}$ defines a topological isomorphism of $D(V)$ onto $D(V)$, hence, in a natural manner (see [5]) $\mathcal{F}$ defines a topological isomorphism $\boldsymbol{F}$ of $\boldsymbol{D}(V)^{\prime}$ onto $\boldsymbol{D}(V)^{\prime}$. $\boldsymbol{F}$ may be regarded as a generalization of the Fourier transform on the space of distributions (see [5]).

In the particular case $V=D_{l}\left(=D_{l}(C)\right)$, we have the following:

Theorem 9. Let $t: C \rightarrow D_{l}$ be an entire map into $D_{l}$ of exponential type $\leqq l$, such that $t$ is rapidly decreasing on $R$. Then we may represent $t$ as an entire function of exponential type $\leqq l$ on $C \times C$ which is rapidly decreasing on $R \times R$.

Proof. We may write, by Theorem $7, t=F(s)$ where $s \in D_{l}\left(D_{l}\right)$. Call $s^{*}$ the map of $C \rightarrow D_{l}$ defined by $\left(s^{*}(z)\right)(x)=(s(x))(z)$. We claim that $s^{*}$ is an entire map of exponential type $\leqq l$ of $C \rightarrow D_{l}$ which is rapidly decreasing on $R$. Assume this is the case; by Theorem 7 we may write $s^{*}=\mathcal{F}(u)$ where $u$ $\in D_{l}\left(D_{l}\right)$. We write $U(x, y)=(u(x))(y)$, so $U$ is an indefinitely differentiable function on $R \times R$ whose carrier is contained in the cube, center origin, side $2 l$. Thus, by the classical Paley-Wiener Theorem (see [5]); the function $T$ defined by 


$$
T(z, w)=\iint U(x, y) e^{-i z x} e^{-i w y} d x d y
$$

is an entire function of exponential on $C \times C$ which is rapidly decreasing on $R \times R$. It is readily verified that $T(z, w)=(t(z))(w)$ for any $(z, w) \in C \times C$.

To conclude the proof, it remains to show that $s^{*}$ is an entire map of exponential type $\leqq l$ which is rapidly decreasing on $R$. Let $W \in D_{l}^{\prime}$; we can find measures $\theta_{0}, \theta_{1}, \cdots, \theta_{r}$ on $R$ whose carriers are contained in $[-l \leqq x \leqq l]$ so that, for any $f \in D_{l}, W \cdot f=\sum \int f^{(j)}(x) d \theta_{j}(x)$. In particular, for any $z \in C$, $W \cdot s^{*}(z)=\sum \int s^{*}(z)^{(j)}(x) d \theta_{j}(x)$. If $\Gamma$ is any closed curve in $C$, then

$$
\begin{aligned}
W \cdot \int_{\Gamma} s^{*}(z) d z & =\sum \int d \theta_{j}(x) \int_{\Gamma} s^{*}(z)^{(j)}(x) d z \\
& =\sum \int d \theta_{j}(x) \int_{\Gamma}\left(s^{(j)}(x)(z) d z\right. \\
& =0
\end{aligned}
$$

because $s^{j}(x)$ is an entire function. Thus, since $s^{*}$ is obviously continuous, $s^{*}$ is analytic.

On the other hand, since $s \in D_{l}\left(D_{l}\right)$, we can find an $M>0$ so that, for all $z$,

$$
\sup _{x \in R, 0 \leqq j \leqq r}\left|\left(s^{(j)}(x)\right)(z)\right| \leqq M \exp (l|z|) .
$$

Thus, by the above,

$$
\begin{aligned}
\left|W \cdot s^{*}(z)\right| & \leqq \sum \int\left|\left(s^{(j)}(x)\right)(z)\right| d\left|\theta_{j}(x)\right| \\
& \leqq M^{\prime} \exp (l|z|)
\end{aligned}
$$

where $M^{\prime}=M(r+1) \max \int d\left|\theta_{j}(x)\right|$.

This shows that $s^{*}$ is an entire map of exponential type $\leqq l$. The fact that $s^{*}$ is rapidly decreasing on $R$ is easily verified. This completes the proof of Theorem 9.

In addition to considering the space $D(V)^{\prime}$, there is yet another way to define vector-valued distributions, namely, by considering the continuous linear maps of $D$ into $V$. We shall show that the two concepts coincide. First we need

Lemma 2. Let us denote by $D \otimes V$ the algebraic tensor product of $D$ and $V$, that is, $D \otimes V$ consists of all finite sums of the form $\sum c_{j} f_{j} \otimes v_{j}$ where $f_{j} \in \mathbb{D}$, $v_{j} \in V$ and $c_{j}$ are complex numbers. We consider $D \otimes V$ as a subspace of $D(V)$ by setting $\left(\sum c_{j} f_{j} \otimes v_{j}\right)(x)=\sum c_{j} f_{j}(x) v_{j}$ for any $x \in R$. Then $D \otimes V$ is dense in $D(V)$.

Proof. Let us define the map $j_{V} \cdot D\left(V^{\prime}\right) \rightarrow \mathscr{D}(V)^{\prime}$ by 


$$
j_{V} h \cdot k=\int[h(x) \cdot k(x)] d x
$$

for any $h \in \mathscr{D}\left(V^{\prime}\right), k \in \mathscr{D}(V)$, where $V^{\prime}$ is the dual of $V$ with the topology of uniform convergence on the bounded sets of $V$. (It follows immediately from the definition of the topology of $\mathscr{D}_{\mathfrak{l}}(V)$ that, for any $h \in \mathbb{D}\left(V^{\prime}\right), j_{V}(h)$ is continuous on $D_{\mathfrak{l}}(V)$; thus, by a result of Dieudonné and Schwartz [2], $j_{V} h$ is continuous on $\mathscr{D}(V)$.) We claim that $j_{V}\left(D\left(V^{\prime}\right)\right)$ is dense in $D(V)^{\prime}$. For, if $B$ is any.bounded set in $D(V)$, then for some $\mathfrak{l}$, all $k \in B$ lie in some $\operatorname{D}_{\mathfrak{l}}(V)$, and $B$ is bounded in $D_{\mathfrak{l}}(V)$ (see [2]). It follows easily from the definition of the topology of $\mathscr{D}_{\mathfrak{l}}(V)$ that, for any $r,\left\{k^{(r)}\right\}_{k \in B}$ are equicontinuous maps of $R \rightarrow V$. Thus, by the Theorem of Ascoli, $B$ has a compact closure in $\mathscr{D}_{\mathfrak{l}}(V)$, hence also in $D(V)$, that is, $D(V)$ is a Montel space. Since $D(V)$ is also bornologic (see [5]), it is reflexive.

Now, suppose $j_{V}\left(D\left(V^{\prime}\right)\right)$ is not dense in $\mathscr{D}(V)^{\prime}$. Then we can find an $L \in \mathscr{D}(V)^{\prime \prime}$ such that $L \neq 0$ and $L \cdot j_{V}\left(\mathscr{D}\left(V^{\prime}\right)\right)=0$. By the reflexivity of $D(V)$, we can find an $h \in \mathscr{D}(V)$ such that $h \neq 0$, and $L \cdot S=S \cdot h$ for all $S \in \mathscr{D}(V)^{\prime}$. In particular, for any $k \in \mathscr{D}\left(V^{\prime}\right)$,

$$
L \cdot j_{V} k=j_{V} k \cdot h=\int[k(x) \cdot h(x)] d x .
$$

Now, let $v^{\prime} \in V^{\prime}$ be so chosen that $v^{\prime} \cdot h(x)$ is not zero for all $x$. (The existence of such a $v^{\prime}$ is guaranteed by the Hahn-Banach Theorem.) We know that $v^{\prime} \cdot h \in D$; thus, we can find an $f \in D$ such that $\int f(x)\left(v^{\prime} \cdot h\right)(x) d x \neq 0$. If we set $k=f \otimes v^{\prime}$ in (5), we have

$$
\begin{aligned}
L \cdot j_{V} k=j_{V} k \cdot h & =\int\left[\left(f \otimes v^{\prime}\right)(x) \cdot h(x)\right] d x \\
& =\int f(x)\left[v^{\prime} \cdot h(x)\right] d x \\
& \neq 0 .
\end{aligned}
$$

This contradicts our assumption that $L=0$ on $j_{V} D\left(V^{\prime}\right)$ and shows that $j_{V}\left(b\left(V^{\prime}\right)\right)$ is dense in $D(V)^{\prime}$.

Before proceeding to our main result, we shall need several preliminary considerations. We shall say that the sequence $\left\{v_{n}^{\prime}\right\}$, where each $v_{n}^{\prime} \in V^{\prime}$, is slowly increasing if there exists a set of continuous semi-norms $\rho_{j}$ on $V^{\prime}$ which are sufficient to define the topology of $V^{\prime}$ such that, for each $j$, we can find an $i$ with

$$
\rho_{j}\left(v_{n}^{\prime}\right)=O\left(1+n^{i}\right) .
$$

The family of sequences $\left\{v_{n}^{\prime}\right\}$ are said to be uniformly slowly increasing if, for each $j$, the symbol $O$ and the number $i$ in (6) can be chosen uniformly for all members of the family. 
LEмma 3. Let $\left\{{v^{\prime \alpha}}_{n}^{\alpha}\right\}_{n=-\infty}^{\infty}$ be uniformly slowly increasing sequences in $V^{\prime}$ and let $\left\{b_{n}\right\}$ be any rapidly decreasing sequence of positive real numbers. Then $\left\{b_{n} v_{n}^{\prime \alpha}\right\}_{n}$, is bounded in $V^{\prime}$.

Proof. This is an immediate consequence of the definitions.

LEMMA 4. Let $\left\{v_{n}^{\beta}\right\}_{n=-\infty}^{\infty}$ be a family of uniformly rapidly decreasing sequences in $V$. Then we can find a rapidly decreasing sequence of positive numbers $\left\{b_{n}\right\}$ such that $\left\{v_{n}^{\beta} / b_{n}\right\}_{n=-\infty}^{\infty}$ are still uniformly rapidly decreasing.

Proof. Let $\left\{\rho_{j}\right\}_{j=1}^{\infty}$ be a sequence of continuous semi-norms on $V$ which are sufficient to define the topology of $V$. (Such a sequence $\left\{\rho_{j}\right\}$ exists because $V$ is metrizable.) For each $j, n$, we set

$$
b_{n}=\left\{\begin{array}{l}
\sup _{\beta}\left[\rho_{j}\left(v_{n}^{\beta}\right)\right]^{1 / 2} \text { if } \rho_{j}\left(v_{n}^{\beta}\right) \neq 0 \text { for some } \beta, \\
n^{-n} \text { otherwise. }
\end{array}\right.
$$

It is clear that $\left\{b_{n}^{j}\right\}_{n=-\infty}^{\infty}$ is a sequence of rapidly decreasing sequences of positive numbers. We extract a new rapidly decreasing sequence of positive numbers $\left\{b_{n}\right\}$ such that, for each $j$, for all but a finite number of $n$ we have $b_{n}^{j} \leqq b_{n}$. The sequence $\left\{b_{n}\right\}$ is defined as follows: Let $a$ be a positive number such that $b_{n}^{1} \leqq a /(1+n)$ for all $n$. Since $b_{n}^{j}=o\left(1 /\left(1+n^{2}\right)\right)$ for all $j$, we can find a positive integer $n_{1}$ such that, for $n \geqq n_{1}$, we have

$$
b_{n}^{1} \leqq \frac{a}{1+n^{2}}, \quad b_{n}^{2} \leqq \frac{a}{1+n^{2}}
$$

More generally, for each $k$ we can find a positive integer $n_{k}$ such that, for $n \geqq n_{k}$,

$$
b_{n}^{j} \leqq \frac{a}{1+n^{k+1}} \quad \text { for } j=1,2, \cdots, k+1
$$

We may obviously assume, in addition, that $n_{k} \rightarrow \infty$; we define $n_{0}=0$.

Now, we set

$$
b_{n}=\frac{a}{1+n^{k}} \quad \text { for } n=n_{k-1}, n_{k-1}+1 \cdots n_{k}-1
$$

Since $n_{k} \rightarrow \infty, b_{n}$ is defined for all integers $n$ and, moreover, $\left\{b_{n}\right\}$ is rapidly decreasing. From (8) and (9) we deduce that, for each $j, b_{n} \geqq b_{n}^{j}$ for all but a finite number of $j$.

Now, it is clear from the definitions that, for each $j,\left\{\rho_{j}\left(v_{n} / b_{n}^{j}\right)\right\}_{n=-\infty}^{\infty}$ are uniformly rapidly decreasing sequences. It follows immediately that $\left\{\rho_{j}\left(v_{n} / b_{n}\right)\right\}_{n=-\infty}^{\infty}$ are also rapidly decreasing sequences uniformly in $V$. This 
means that $\left\{v_{n} / b_{n}\right\}$ are uniformly rapidly decreasing sequences. This concludes the proof of Lemma 4.

LEMMA 5. Let $\left\{v_{n}^{\prime \alpha}\right\}_{n=-\infty}^{\infty}$ be uniformly slowly increasing sequences in $V^{\prime}$, and let $\left\{v_{n}^{\beta}\right\}_{n=-\infty}^{\infty}$ be uniformly rapidly decreasing sequences in $V$. Then we can find an $M>0$ so that $\sum\left|v_{n}^{\prime \alpha} \cdot v_{n}^{\beta}\right| \leqq M$ for all $\alpha, \beta$.

Proof. By Lemma 4 , we can find a rapidly decreasing sequence $\left\{b_{n}\right\}$ of positive numbers such that $\left\{\left(1+n^{2}\right) v_{n}^{\beta} / b_{n}\right\}_{n, s}$ is bounded in $V$. By Lemma 3 , we know that $\left\{b_{n} v_{n}^{\prime \alpha}\right\}_{n, \alpha}$ is bounded in $V^{\prime}$. Thus, we can find a constant $M^{\prime}>0$ such that, for all $n, \alpha, \beta$, we have

$$
M^{\prime} \geqq\left|b_{n} v_{n}^{\prime} \cdot \frac{\left(1+n^{2}\right) v_{n}}{b_{n}}\right|=\left(1+n^{2}\right)\left|v_{n}^{\prime \alpha} \cdot v_{n}^{\beta}\right| .
$$

It follows that $\sum_{n}\left|v_{n}^{\prime \alpha} \cdot v_{n}^{\beta}\right| \leqq M^{\prime} \sum 1 /\left(1+n^{2}\right)$ for all $\alpha, \beta$, which gives the desired result with $M=M^{\prime} \sum 1 /\left(1+n^{2}\right)$.

Let $f \in D(V)$; assume the carrier of $f$ is contained in the interval $[\mathfrak{l}+1 / 8$ $\leqq x \leqq \mathfrak{l}+7 / 8]$. Denote by $f^{*}$ the map of $R$ into $V$ which is equal to $f$ in $[\mathfrak{l} \leqq x \leqq \mathfrak{l}+2 \pi]$ and periodic of period $2 \pi$, i.e. $f^{*}(x+2 \pi)=f^{*}(x)$ for all $x \in R$. We define the Fourier coefficients $f_{n}^{*} \in V$ of $f^{*}$ for any integer $n$ by

$$
f_{n}^{*}=\int_{0}^{2 \pi} f^{*}(x) \exp (-i n x) d x .
$$

The following proposition sums up the properties of the $f_{n}^{*}$ which we shall need:

Proposition 15. Let $B$ be a bounded set in $\mathscr{D}_{\mathfrak{l}}(V)$; then the sequences $\left\{f_{n}^{*}\right\}$ are rapidly decreasing in $V$ uniformly for $f \in B$, i.e. for any continuous seminorm $\dot{\rho}$ on $V$ and any $j, \rho\left(f_{n}^{*}\right)=O\left(1 /\left(1+n^{j}\right)\right)$ uniformly for $f \in B$. The series $\sum f_{n}^{*} \exp ($ inx $)$ converge to $f^{*}(x)$ in $V$ uniformly for $f \in B, x \in R$. If $f^{k} \rightarrow 0$ in $D_{I}$, then for any continuous semi-norm $\rho$ on $V$, and any $j,\left(1+n^{j}\right) \rho\left(f_{n}^{* k}\right) \rightarrow 0$.

Proof. The fact that the sequences $\left\{f_{n}^{*}\right\}$ are uniformly rapidly decreasing for $f \in B$ is easily verified by standard methods in the theory of Fourier series. (See e.g. [7, Chapter VII, §1], where the result is proved for numerical valued functions; the result for vector valued functions is proved similarly.) From this it follows that the series $\sum f_{n}^{*} \exp (i n x)$ converge in $V$ uniformly for $f \in B, x \in R$. Now, for any $v^{\prime} \in V^{\prime}$,

$$
v^{\prime} \cdot f_{n}^{*}=\int_{0}^{2 \pi}\left[v^{\prime} \cdot f^{*}(x)\right] \exp (-i n x) d x
$$

so that $v^{\prime} \cdot f_{n}^{*}$ are the Fourier coefficients (in the usual sense) of the function $x \rightarrow v^{\prime} \cdot f^{*}(x)$. Thus,

$$
v^{\prime} \cdot \sum f_{n}^{*} \exp (i n x)=\sum\left(v^{\prime} \cdot f_{n}^{*}\right) \exp (i n x)=v^{\prime} \cdot f^{*}(x)
$$


by a classical theorem on Fourier series. Thus, by the Hahn-Banach theorem, $\sum f_{n}^{*} \exp ($ inx $)$ converges in $V$ to $f^{*}(x)$ uniformly for $x \in R, f \in B$. The last part of Proposition 15 is easily verified by standard methods.

Proposition 16. Let $f \in D(V)$, and suppose that the carrier of $f$ is contained in $[\mathfrak{l}+1 / 8 \leqq x \leqq \mathfrak{l}+7 / 8]$. Then we may write

$$
f=\sum f_{n}^{1} \otimes v_{n}
$$

where $\left\{f_{n}^{1}\right\}$ is slowly increasing in $D$ and $\left\{v_{n}\right\}$ is rapidly decreasing in $V$, and where the series converges in $D(V)$. The $f_{n}$ are independent of $f$ and the $v_{n}$ depend linearly on $f$. If $B$ is a bounded set in $D(V)$, then we may suppose that the sequences $\left\{v_{n}\right\}$ are uniformly rapidly decreasing for $f \in B$. If $f^{k} \rightarrow 0$ in $D_{\mathfrak{l}}(V)$, then we may write

$$
f^{k}=\sum f_{n}^{\mathfrak{l}} \otimes v_{n}^{k}
$$

in such a manner that for any $r>0,\left(1+n^{r}\right) v_{n}^{\boldsymbol{k}} \rightarrow 0$ in $V$ uniformly in $n$.

Proof. Let $f^{*}$ have the same meaning as in Proposition 15. Let $t \in \mathcal{D}$ satisfy

$$
t(x)=\begin{aligned}
& 1 \text { for } \mathfrak{l}+1 / 8 \leqq x \leqq \mathfrak{l}+7 / 8, \\
& 0 \text { for } x \leqq \mathfrak{l}, \text { or for } x \geqq \mathfrak{l}+1 .
\end{aligned}
$$

By Proposition 15 we have, for any $x \in R$,

$$
f(x)=\sum t(x) \exp (i n x) f_{n}^{*}(x)
$$

where the series converges in $V$ uniformly for $x \in R$. The result follows from Proposition 15 and Lemma 5 on setting $f_{n}^{l}=t \exp (i n x), v_{n}=f_{n}^{*}$.

For any $S \in \mathbb{D}(V)^{\prime}, f \in \mathbb{D}, v \in V$, we define $\lambda S(f) \in V^{\prime}$ by

$$
\lambda S(f) \cdot v=S \cdot f \otimes v .
$$

Let us denote by $Q$ the space of continuous linear maps of $D \rightarrow V^{\prime}$ with the compact-open topology. Then our main result is:

TheOREM 10. $\lambda$ defines a topological isomorphism of $D(V)^{\prime}$ onto $Q$.

Proof. It is easily verified that $\lambda$ is a linear map. From the above Lemma $2, \lambda$ is one-one. To show that $\lambda$ is continuous, we must show the following: Let $\left\{S_{\alpha}\right\}$ be a filter base in $D(V)^{\prime}$ which $\rightarrow 0$ in $D(V)^{\prime}$, and let $B$ be a bounded set in $D$; then $\lambda S_{\alpha}(f) \rightarrow 0$ in $V^{\prime}$ uniformly for $f \in B$. By the definition of the topology of a dual space, this is the same as saying that, for $A$ a bounded set in $V$,

$$
\lambda S_{\alpha}(f) \cdot v=S_{\alpha} \cdot f \otimes v \rightarrow 0
$$

uniformly for $f \in B, v \in A$. But, it is easily verified that $\{f \otimes v\}_{f \in B, v \in A}$ is 
bounded in $\mathscr{D}(V)$. Thus, (15) is established, which implies the continuity of $\lambda$. Let $\left\{g_{j}\right\}_{j=-\infty}^{\infty}$ be a sequence of functions in D such that, for each $j$, carrier $g_{j} \subset[j+1 / 8 \leqq x \leqq j+7 / 8]$, and for any $x \in R, \sum g_{j}(x)=1$. Given any $h \in \mathcal{D}(V)$, we shall set $h_{j}=g_{j} h$, i.e. $h_{j}(x)=g_{j}(x) h(x)$ for any $x \in R$. It is clear that $h_{j} \in \mathscr{D}(V)$ for each $j$, and that $h=\sum h_{j}$ where only a finite number of terms in the series are different from zero.

By Proposition 16, we may write each $h_{j}$ in the form

$$
h_{j}=\sum f_{n}^{j} \otimes v_{n}^{j}
$$

where $\left\{v_{n}^{j}\right\}_{n=-\infty}^{\infty}$ is rapidly decreasing in $V$ and $\left\{f_{n}^{j}\right\}_{n=-\infty}^{\infty}$ is slowly increasing in $D$. Moreover, $f_{n}^{j}$ depends only on $n$ and $j$, and not on $h$, and the $v_{n}^{j}$ depend linearly on $h$.

Now, given any $T \in Q$, we define $\gamma T \in D^{\prime}$ by

$$
\gamma T \cdot h=\sum_{j} \sum_{n=-\infty}^{\infty} T f_{n}^{j} \cdot v_{n}^{j}
$$

for any $h \in \mathbb{D}(V)$. We must show that the series on the right side of (17) converges and defines $\gamma T$ as an element of $D(V)^{\prime}$. Now, since $T \in Q$, given any continuous semi-norm $\rho$ on $V^{\prime}$ of the kind used to define the topology of $V^{\prime}$, we can find a neighborhood $N$ of zero in $D$ such that, for any $f \in N, \rho(T f) \leqq 1$. Since $\left\{f_{n}^{j}\right\}_{n=-\infty}^{\infty}$ is slowly increasing, we can find a slowly increasing sequence $\left\{a_{n}^{j}\right\}$ of positive numbers such that $f_{n}^{j} / a_{n}^{j} \in N$ for all $n$. Thus, $\rho\left(T f_{n}^{j}\right) \leqq a_{n}^{j}$, i.e. $\left\{T f_{n}^{j}\right\}$ is slowly increasing in $V^{\prime}$. Since $\left\{v_{n}^{j}\right\}$ is rapidly decreasing in $V$, by Lemma $5, \sum_{n=-\infty}^{\infty} T f_{n}^{j} \cdot v_{n}^{j}$ converges absolutely. Since only a finite number of $j$ appear in (17), it follows that the series on the right side of (17) converges absolutely.

Now, $f_{n}^{j}$ is independent of $h$, and the $v_{n}^{j}$ depend linearly on $h$. It follows immediately that $\gamma T$ is a linear function on $D(V)$. By reasoning exactly as above, and using the fact that, for $B$ a bounded set in $D(V)$, all $h \in B$ have their carriers in a fixed compact set of $R$, we deduce that $\gamma T$ is bounded on the bounded sets of $D(V)$. Thus, by a result of Dieudonné and Schwartz [2], $\gamma T \in D(V)^{\prime}$.

We claim that, for any $T \in Q, \lambda \gamma T=T$. For, given any $f \in D, v \in V$,

$$
\lambda \gamma T(f) \cdot v=\gamma(T) \cdot f \otimes v .
$$

If we set $h=f \otimes v$, then $h_{j}=g_{j} f \otimes v$. It follows easily from the definitions that (16) becomes

$$
h_{j}=g_{j} f \otimes v=\sum_{n} f_{n}^{j} \otimes e_{n}^{j} v
$$

where $\left\{e_{n}^{j}\right\}_{n=-\infty}^{\infty}$ is a rapidly decreasing sequence of constants. Thus, (17) becomes 


$$
\begin{aligned}
\gamma T \cdot h & =\gamma T \cdot f \otimes v=\sum_{j} \sum_{n} T f_{n}^{j} \cdot e_{n}^{j} v \\
& =\sum_{j} \sum_{n} T e_{n}^{j} f_{n}^{j} \cdot v \\
& =\left[T \sum_{j, n} e_{n}^{j} f_{n}^{j}\right] \cdot v \\
& =T f \cdot v
\end{aligned}
$$

because, as is easily seen, the series $\sum_{j, n} e_{n}^{j} f_{n}^{j}$ converges in $D$ to $f$. This proves that $\lambda \gamma T=T$.

From the above, it results that $\lambda$ is onto. Moreover, $\lambda \gamma=$ identity, so $\lambda \gamma \lambda=\lambda$; since $\lambda$ is one-one and onto, this shows that $\gamma \lambda=$ identity. Thus, $\gamma$ is a two sided inverse for $\lambda$.

To complete the proof of Thereom 10, it remains to show that $\gamma$ is continuous. It is obvious that $\gamma$ is linear, so we have to prove continuity only at zero. Let $T^{\alpha} \rightarrow 0$ in $Q$, and let $B$ be a bounded set in $D(V)$; we must show that $\gamma T^{\alpha} \cdot h \rightarrow 0$ uniformly for $h \in B$. By Lemma 4 and Proposition 16 , we can find a rapidly decreasing sequence $\left\{b_{n}^{j}\right\}$ of positive numbers such that, for any $j$, the sequences $\left\{v_{n}^{j} / b_{n}^{j}\right\}_{n=-\infty}^{\infty}$ as defined by (16) are stilt uniformly rapidly decreasing in $V$ for $h \in B$. By Lemma 3 and Proposition 16, for each $j$, $\left\{b_{n}^{j} f_{n}^{j}\right\}_{n=-\infty}^{\infty}$ is bounded in $D$. Thus, by the definition of the topology of $Q$, for each $j, T^{\alpha}\left(b_{n}^{j} f_{n}^{j}\right) \rightarrow 0$ in $V^{\prime}$ uniformly in $n$. This means that, for each $j$,

$$
\sum_{n}\left|T^{\alpha} f_{n}^{j} \cdot v_{n}^{j}\right|=\sum_{n}\left|T^{\alpha}\left(b_{n}^{j} f_{n}^{j}\right) \cdot \frac{v_{n}^{j}}{b_{n}^{j}}\right| \rightarrow 0
$$

uniformly for $h \in B$. Since only a finite number of $j$ are sufficient for all $h \in B$, it follows that

$$
\gamma T^{\alpha} \cdot h \rightarrow 0 \text { uniformly for } h \in B .
$$

This proves the continuity of $\gamma$ and concludes the proof of Theorem 10 .

REMARK 1. Theorem 10 is the general theorem on kernels of L. Schwartz (see A. Grothendieck, Products tensoriels topologiques et espaces nucléaires, Memoirs Amer. Math. Soc., no. 16, 1955; see also my paper On the theory of kernels of Schwartz, Proc. Amer. Math. Soc. vol. 7 (1956) pp. 713-718 where the special case $V=\mathbb{D}$ is treated by a method similar to the above).

REMARK 2. Most of the above may be carried out if $V$ is assumed to be complete, but not necessarily metrizable. In that case, however, $D(V)$ is not an $(L F)$ space in the sense of Dieudonné and Schwartz. If $V$ is not metrizable, then the map $\gamma$ can only be proved to be sequentially continuous or, more generally, if $\beta$ is a filter base of bounded sets of $Q$ which $\rightarrow 0$ in $Q$, then $\gamma(\beta) \rightarrow 0$ in $\mathscr{D}(V)^{\prime}$. The continuity of $\gamma$ in general is probably false; we have not been able to construct a counter example. 
REMARK 3. Theorem 10 and its proof can be easily extended to the case in which the real line is replaced by any separable indefinitely differentiable manifold or double coset space of a Lie group.

7. Functions analytic in a strip. In this section we lay the groundwork for the application of the theory of distributions to classical analytic function theory. We study here the properties of functions analytic in a strip because Fourier analysis seems especially amenable to such functions; corresponding results for other regions may be obtained easily by conformal mapping. We shall assume in this section that $n=1$ although it is not difficult to extend most of our methods to the general case.

Proposition 1. Let $H$ and $K$ be functions on $R$ for which $\mathrm{e}(H)$ and $\mathrm{e}(K)$ are defined. Suppose $\mathrm{e}(H)=\mathfrak{e}(K)$; then for almost every $x \in R, H(x)=K(x)$.

Proof. Let $F \in D, F \neq 0$; then $F H$ and $F K$ are integrable functions. Clearly, for every real $t, F H \exp (i t \cdot)$ and $F K \exp (i t)$ are also integrable. Now, since $F \exp (i t \cdot) \in D$,

$$
\mathfrak{e}(H) \cdot F \exp (i t \cdot)=\mathfrak{e}(K) \cdot F \exp (i t \cdot)
$$

or

$$
\int \bar{H}(x) F(x) \exp (i t x) d x=\int \bar{K}(x) F(x) \exp (i t x) d x
$$

for every $t \in R$. Thus, the classical Fourier transforms of $\bar{H} F$ and $\bar{K} F$ are identical. Hence (see [8]) for almost every $x, \bar{H}(x) F(x)=\bar{K}(x) F(x)$. Since $F$ is an entire function different from zero, the result follows.

If $b$ is any complex number and $A$ a function defined on a subset of $C$, then $\tau_{b} A$ is the function $\left(\tau_{b} A\right)(z)=A(z-b)$ for $z$ in an appropriate subset of $C$.

Let $a<b$ be real numbers, or $a=-\infty$, or $b=+\infty$. We shall say that the function $A$ satisfies property $L[a, b]$ if

(a) $\bar{A}$ is analytic in the strip

$$
a<\mathfrak{g}(z)<b
$$

(b) For any $c$ in the strip (1), $\mathrm{e}\left(\tau_{c} A\right)$ is defined.

(c) For any $G \in D$, the integrals $\int_{R+i d}|A(x) G(x)| d x$ are bounded uniformly for $a<a^{\prime} \leqq d \leqq b^{\prime}<b$.

Theorem 1. Let $A$ satisfy property $L[a, b]$. Then for any $G \in D$ and any $c, d$ in the strip (1),

$$
\begin{aligned}
\int_{R+c} \bar{A}(z) G(z) d z & =\int_{R+d} \bar{A}(z) G(z) d z . \\
\mathfrak{e}\left(\tau_{-d} A\right) & =\tau_{c-d} \mathfrak{e}\left(\tau_{-c} A\right) .
\end{aligned}
$$

Proof. It is clearly sufficient to consider the case where $c$ and $d$ lie on the 
imaginary axis, say $c=i c^{\prime}, d=i d^{\prime}$ where $c^{\prime}$ and $d^{\prime}$ are real; we assume that $c^{\prime}<d^{\prime}$, the other case being treated similarly. Call $B=\bar{A} G$, so $B$ is analytic in the strip (1). Let $\rho$ be any positive number such that

$$
\left.\rho \leqq \min \left(\left(b-d^{\prime}\right) / 2,\left(c^{\prime}-a\right) / 2\right)\right) .
$$

Then for any $z$ satisfying $a+\rho<\mathfrak{g}(z)<b-\rho$, by Cauchy's formula,

$$
\begin{aligned}
B(z) & =\frac{1}{i} \int_{|w-z|=\rho} \frac{B(w)}{w-z} d w \\
& =\int_{0}^{2 \pi} B\left(z+\rho e^{i \theta}\right) d \theta .
\end{aligned}
$$

Thus,

$$
\begin{aligned}
\left|\int_{c^{\prime}}^{d^{\prime}} B(x+i y) d y\right| & =\left|\int_{c^{\prime}}^{d^{\prime}} d y \int_{0}^{2 \pi} B\left(x+i y+\rho e^{i \theta}\right) d \theta\right| \\
& =\left|\int_{0}^{2 \pi} d \theta \int_{c^{\prime}}^{d^{\prime}} B\left(x+i y+\rho e^{i \theta}\right) d y\right| \\
& \leqq \int_{c^{\prime}-\rho}^{d^{\prime}+\rho} d y \int_{x-\rho}^{x+\rho}|B(t+i y)| d t .
\end{aligned}
$$

The functions $F_{x}$ defined by

$$
F_{x}(y)=\int_{x-\rho}^{x+\rho}|B(t+i y)| d t
$$

are continuous, converge pointwise to zero as $x \rightarrow \infty$, and are uniformly bounded, because $A$ has property $L[a, b]$. Thus, by the Lebesgue convergence criterion,

$$
\int_{c^{\prime}-\rho}^{d^{\prime}+\rho} F_{x}(y) d y \rightarrow 0
$$

as $x \rightarrow \infty$. Equation (2) is an immediate consequence of the above and Cauchy's theorem.

Formula (3) follows immediately from formula (2) and the definitions.

The next proposition may be regarded, in a certain sense, as an extension of Morera's theorem.

Proposition 2. Let $a<b$ be real numbers or $a=-\infty$ or $b=+\infty$. Let $S \in D^{\prime}$ have the following property: there exist sequences of real numbers $a_{k}>a$ and $b_{j}<b$ such that $a_{k}>a$ and $b_{j}>b$, and for each $j, k$ there are functions $F_{j}, G_{k}$ for which $\mathrm{e}\left(\tau_{-i a_{k}} G_{k}\right)$ and $\mathrm{e}\left(\tau_{i b_{j}} F_{j}\right)$ are defined and equal respectively to $\tau_{-i a_{k}} S$, $\tau_{-i b j} S$. Then there exists a function $A$ which satisfies property $L[a, b]$ and such that 


$$
\mathfrak{e}\left(\tau_{-c} A\right)=\tau_{-\sigma} S
$$

for any $c$ in the strip (1). In particular, for any $j$,

$$
\tau_{-i b_{j}} A=\tau_{-i b_{j}} F_{j} \quad \text { a.e. }
$$

and, for any $k$,

$$
\tau_{-i a_{k}} A=\tau_{-i a_{k}} G_{k} \quad \text { a.e. }
$$

Proof. Let $K \in D$; for any $t \in R$; $\exp (i t \cdot) K$ is again in $D$. Set

$$
h(t)=\mathrm{e}\left(\tau_{-i a_{1}} G_{1}\right) \cdot K \exp (i t \cdot)
$$

Then for any $k$,

$$
\begin{aligned}
h(t) & =e\left(\tau_{-i a_{1}} G_{1}\right) \cdot K \exp (i t \cdot) \\
& =\tau_{-i a_{1}} S \cdot K \exp (i t \cdot) \\
& =\tau_{i\left(a_{k}-a_{1}\right)} \tau_{-i a_{k}} S \cdot K \exp (i t \cdot) \\
& =\tau_{-i a_{k}} S \cdot \tau_{i\left(a_{1}-a_{k}\right)} K \exp (i t \cdot) \\
& =\tau_{-i a_{k}} S \cdot \tau_{i\left(a_{1}-a_{k}\right)} K \tau_{i\left(a_{1}-a_{k}\right)} \exp (i t \cdot) \\
& =e^{\left(a_{1}-a_{k}\right) t_{-i a_{k}} S \cdot \tau_{i\left(a_{1}-a_{k}\right)} K \exp (i t \cdot)} \\
& =e^{\left(a_{1}-a_{k}\right) t} \int \bar{G}_{k}\left(x+i a_{k}\right) K\left(x+i a_{k}-i a_{1}\right) e^{i t x} d x .
\end{aligned}
$$

Thus,

$$
e^{\left(a_{k}-a_{1}\right) t} h(t)=\int \bar{G}_{k}\left(x+i a_{k}\right) K\left(x+i a_{k}-i a_{1}\right) e^{i t x} d x .
$$

According to our hypotheses, the right side of (4) is bounded independently of $t$. We conclude that $e^{\left(a_{k}-a_{1}\right) t} h(t)$ is bounded independently of $t$. By similar reasoning, we can show that the same is true of the functions $\exp \left(b_{j}-a_{1} \cdot\right) h$ for any $j$. Denote by $H$ the classical Fourier transform of $h$. Then the above shows us that $H$ is analytic in the strip $a-a_{1}<g(z)<b-a_{1}$ and is bounded uniformly in any closed substrip. Now, by the classical Fourier inversion formula, $H=K \tau_{-i a_{1}} \bar{G}_{1}$ almost everywhere on $R$. Thus,

$$
\tau_{i a_{1}} H=\bar{G}_{1} \tau_{i a_{1}} K \quad \text { a.e. }
$$

can be extended to a function which is analytic in the strip (1). Since $K$ was chosen arbitrarily in $D$, this means that there exists a function $\bar{A}$ which is analytic in (1) and which is equal to $\bar{G}_{1}$ almost everywhere in $R$.

From the above it follows immediately that, if $J$ is any element of $D$, $A J$ is bounded in any closed substrip of (1). Since $Z^{2} J \in D$, it follows that $Z^{2} A J$ is bounded in any closed substrip of (1). ( $Z$ is the function on $C: z \rightarrow z$.) From this it follows immediately that $A$ satisfies property $L[a, b]$. 
From our hypotheses, $\mathrm{e}\left(\tau_{-i a_{1}} A\right)=\tau_{-i a_{1}} S$. Combining this with Theorem 1, it follows that $\mathrm{e}\left(\tau_{-c} A\right)=\tau_{-c} S$ for any $c$ in (1). It follows from Proposition 1 that, for each $j, \tau_{-i b_{j}} A=\tau_{-i b_{j}} F_{j}$ a.e., and for each $k, \tau_{-i a_{k}} A=\tau_{-i a_{k}} G_{k}$ a.e.

Corollary. Let $F$ be continuous in (1) and $S \in D^{\prime}$. Suppose that for each $c$ in (1), $\mathfrak{e}\left(\tau_{-c} F\right)$ is defined and is equal to $\tau_{-c} S$. Then $F$ satisfies property $L[a, b]$.

Proposition 3. Let $S \in E^{\prime}$. Then we can find an entire function of exponential type $G$ such that $\bar{G}$ satisfies property $L[-\infty, \infty]$ and such that $S=\mathfrak{e}(\bar{G})$.

Proof. We know (see [6]) that we can write

$$
S=\sum_{j=1}^{r} \mathrm{e}\left(P_{j} \bar{M}_{j}\right)
$$

where $P_{j}$ are polynomials and $M_{j}$ are entire functions of exponential type which are bounded on the real axis. By the Phragmen-Lindelof theorem (see [4]) for some $\mathfrak{l}>0$ the functions $\exp (i \mathfrak{l} \cdot) M_{j}$ are bounded in the upper half plane, and the functions exp $(-i \mathfrak{l} \cdot) M$, are bounded in the lower half plane.

Call $G=\sum P_{j} M_{j}$; it follows from the above that, for any $c>0$, we can find constants $h, k$ such that

$$
|G(x+i y)| \leqq k\left(1+|x|^{h}\right)
$$

for $-c \leqq y \leqq c$. It is immediate that $G$ has property $L[-\infty, \infty]$.

Let $F$ be a function in the strip (1) such that, for any $z$ in that strip, $\mathfrak{e}\left(\tau_{-a} F\right)$ is defined. We say that $S \in D^{\prime}$ is the boundary value of $F$ at $a$ if $\lim _{d \rightarrow 0} \mathrm{e}\left(\tau_{-i a-i d} F\right)=S$. (A similar definition is given for boundary value at $b$.) We assume in the definition that $a \neq-\infty$ (or that $b \neq+\infty$ ).

Proposition 4. Let $F$ have property $L[a, b]$ where $a \neq-\infty$. Suppose that $S \in D^{\prime}$ is the boundary value of $F$ at a. Then for any $c$ in the strip (1),

$$
\tau_{-a} S=e\left(\tau_{-i a-c} F\right) .
$$

Proof. We showed in $\S 2$ that $z \rightarrow \tau_{2} S$ is a continuous map of $C$ into $D^{\prime}$. Thus,

$$
\begin{aligned}
\tau_{-a} S & =\tau_{-c} \lim _{d \rightarrow 0} \mathrm{e}\left(\tau_{-i a-i d} F\right) \\
& =\lim _{d \rightarrow 0} \tau_{-c} \mathrm{e}\left(\tau_{-i a-i d} F\right) \\
& =\lim _{d \rightarrow 0} \mathrm{e}\left(\tau_{-i a-i d-c} F\right) \quad \text { by Theorem 1) } \\
& =\lim _{d \rightarrow 0} \tau_{-i d} \mathfrak{e}\left(\tau_{-i a-c} F\right) \quad \text { (by Theorem 1) } \\
& =\mathfrak{e}\left(\tau_{-i a-c} F\right)
\end{aligned}
$$


by the above-mentioned continuity of translation.

From Propositions 1 and 4 we have

Corollary. Let $A$ and $B$ both saitisfy property $L[a, b]$, where $a \neq-\infty$. Suppose that $A$ and $B$ both have the same boundary values at $a$. Then $A=B$.

Proposition 5. Let $F$ satisfy property $L[a, b]$ where $a \neq-\infty$. Then there is an $S \in D^{\prime}$ which is the boundary value of $F$ at a.

Proof. Let $a<d<b$, and set $T=F^{-1} \mathfrak{e}\left(\tau_{-i d} F\right)$. We claim that

$$
S=F \exp (d-a \cdot) T
$$

is the boundary value of $F$ at $a$. We have, for any $a<c<b$,

$$
\begin{aligned}
\mathfrak{e}\left(\tau_{-i c} F\right) & =\mathfrak{e}\left(\tau_{i(d-c)} \tau_{-i d} F\right) \\
& =\tau_{i(d-c)} \mathfrak{e}\left(\tau_{-i d} F\right) \quad(\text { by Theorem } 1) \\
& =F \exp (d-c \cdot) T \\
& =F \exp (a-c \cdot) \exp (d-a \cdot) T \\
& =\tau_{i(a-c)} S .
\end{aligned}
$$

The proposition results immediately from the above and the continuity of translation.

The above proposition shows us why it is often inconvenient to allow $a=-\infty$ (or $b=+\infty$ ) when considering boundary values: Let $A$ be a function which is defined in a strip $-\infty<I(z)<b$, and suppose that for each $c$ in this strip, $\mathfrak{e}\left(\tau_{-c} A\right)$ is defined. Then we would expect to define $S \in D^{\prime}$ as the boundary value of $A$ at $-\infty$ if $\lim _{d \rightarrow \infty} \mathfrak{e}\left(\tau_{i d} A\right)=S$. But then Proposition 5 is false if we allow $a=-\infty$ as the example $\bar{Z}$, which satisfies property $L[-\infty, \infty]$ shows.

Proposition 6. Let $a<c<b$. Let $F$ satisfy property $L[c, b]$ and let $G$ satisfy property $L[a, c]$. Suppose the boundary values of $F$ and $G$ at $c$ are equal. Then there is a function $H$ which satisfies property $L[a, b]$ and which is an analytic continuation of $F$ and $G$.

Proof. This is an immediate of Propositions 2 and 4.

Let $B$ be a subspace of $D^{\prime}$. We shall say that $B$ has property $M$ if

(1) $B$ is contained in the space $\mathcal{S}^{\prime}$ of Schwartz (see [6]).

(2) $B$ is a module over the space $S$ of Schwartz under convolution, that is, for each $T \in B, F \in \mathcal{S}, F * T \in B$. (This convolution has meaning since $\mathcal{S}$ is clearly a subspace of $E$.)

(3) For each $T \in B, F \rightarrow F * T$ is a continuous map of $\delta$ into $B$.

Let us denote by $B$ the space of inverse Fourier transforms of $B$ with the topology to make the Fourier transform (restricted to $B$ ) a topological isomorphism of $B$ onto $B$. Then $B$ has property $M$ if and only if 
$\left(1^{\prime}\right) Q$ is contained in the space $S^{\prime}$.

$\left(2^{\prime}\right) \Theta$ is a module over $\delta$ under multiplication, that is, for $T \in \leftrightarrow, f \in \delta$, $f T \in B$.

$\left(3^{\prime}\right)$ For each $T \in B, f \rightarrow f T$ is a continuous map of $S$ into $B$.

If $V$ is a topological vector space of equivalence classes of locally summable functions (two functions being identified if and only if they are equal a.e.) then we denote by $i V$ the space of if for $f \in V$ with the topology to make $\dot{i}$ a topological anti-isomorphism ( $\mathfrak{i}$ is clearly one-one). If, for each $f \in V$, ef is defined, then we define $\mathrm{e} V$ as the space of ef for $f \in V$ with the topology to make $e$ a topological anti-isomorphism.

For $1 \leqq p \leqq \infty$, we denote by $L_{p}$ the space of functions on $R$ whose $p$ th powers are integrable; $L_{p}$ being given the usual norm. It is clear that each $\mathrm{e} L_{p}$ has property $M$. Denote by $\mathscr{L}_{p}$ the space of Fourier transforms of $\mathrm{i} L_{p}$ with the topology to make the Fourier transform a topological isomorphism. Thus, $\mathscr{L}_{2}=e L_{2}$ and $\mathscr{L}_{p} \subset e L_{p^{\prime}}$ for $1 \leqq p \leqq 2$, where $1 / p^{\prime}+1 / p=1$. For $p>2$ we do not know whether each $T \in \mathfrak{L}_{p}$ can be represented in the form e $F$ where $F$ is a locally summable function on $R$. Since the spaces $\dot{i} L_{p}$ clearly satisfy conditions $\left(1^{\prime}\right),\left(2^{\prime}\right)$, and $\left(3^{\prime}\right)$ above, the spaces $\mathscr{L}_{p}$ also satisfy property $M$.

Other examples of spaces which satisfy property $M$ are the spaces $\delta, \boldsymbol{s}^{\prime}$, $O_{C}^{\prime}$ of Schwartz (see [6]), and the spaces $\mathcal{E}^{\prime}, E^{\prime}$.

We come now to our extension of the following theorem of Phragmen and Lindelof: Let $F$ be analytic in the open strip $a<g(z)<b$ and continuous in a closed strip. Suppose that $\tau_{-i a} F$ and $\tau_{-i b} F$ are bounded functions and that $F$ does not "grow too fast" at infinity in this strip. Then $F$ is bounded uniformly in this strip. (See [7] for the precise conditions of the theorem.) Now, the fact that $\tau_{-i a} F$ and $\tau_{-i b} F$ are bounded means they are in $L_{\infty}$. Suppose instead of $\tau_{-i a} F$ and $\tau_{-i b} F$ being in $L_{\infty}$ that they are in some $L_{p}$ space or, more generally, if $\mathfrak{e}\left(\tau_{-i a} F\right)$ and $\mathrm{e}\left(\tau_{-i b} F\right)$ are in some space $V$ which satisfies property $M$. Then we shall show that, if $a<c<b, \mathrm{e}\left(\tau_{-i c} F\right) \in M$ and the set $\left\{\tau_{-i d} F\right\}_{a^{\prime} \leq d \leq b^{\prime}}$ is bounded in $V$ whenever $a<a^{\prime}<b^{\prime}<b$.

Lemma. Let $h \in S$ vanish for all $x \leqq c$ for some $c \in R$. Suppose also that there is an $a>0$ so that, for any $k$,

Then

$$
\left(\frac{d^{k} h}{d X^{k}}\right)(x)=O(\exp (-a x))
$$
a. $H$ is analytic in the strip $-\infty<g(z)<a$.
b. For any $z$ in this strip, $\tau_{-z} H \in \mathcal{S}$.
c. Whenever $c<d<a$, the set $\left\{\tau_{-i e} H\right\}_{c \leqq s}$ is bounded in $\mathrm{S}$.

Proof. Statement a is obvious. Part b follows from the fact that $\tau_{z} H$ is the Fourier transform of $\exp (i z \cdot) h$ which is clearly in $\delta$ for any $z$ with $-\infty<g(z)<a$. Part $c$ follows from the obvious fact that $\{\exp (-i e \cdot)\}_{c \text { sesd }}$ is bounded in $S$. 
Proposition 7. Let $h$ satisfy the above hypotheses and let $T \in \mathcal{S}^{\prime}$. Then the Fourier transform of $h T$ can be represented as $\mathrm{e}(k)$ where $K$ satisfies property $L[-\infty, a]$. (We consider $h \in E$ and $T \in D^{\prime}$.)

Proof. Let $S$ denote the Fourier transform of $T$, so also $S \in \mathcal{S}$. It is known (see [6]) that we may write

$$
F(h S)=H * S=\mathrm{e}(S * H)
$$

where $S * H$ is the function on $R$ :

$$
(S * H)(x)=\bar{S} \cdot \tau_{x} H
$$

and $S * H$ is in the space $O_{M}$ of Schwartz.

Now, we may write $S$ in the form $S=\left(d^{r} / d X^{r}\right)^{\prime} \mathrm{e}(G)$ where $G$ is a slowly increasing continuous function on $R$ (see [6]). For any $z$ with $-\infty<g(z)<a$, define

$$
K(z)=\int G(x) H^{(r)}(z-x) d x
$$

In virtue of the above lemma, the integrals converge uniformly for $z$ in any compact subset of $-\infty<I(z)<a$ so that $K$ is analytic in this strip. Moreover, $K(x)=(S * H)(x)$ for $x \in R$. Finally, let $|G(x)| \leqq \alpha_{1}\left(1+|x|^{t}\right)$ and

$$
\left|H^{(r)}(z-x)\right| \leqq \alpha_{2}\left(1+|z-x|^{t+2}\right)^{-1}
$$

for $b \leqq I(z) \leqq d$, where $b<d<a$, and where $t, \alpha_{1}$ and $\alpha_{2}$ are constants. (The existence of $\alpha_{2}$ is assured by the above lemma.) Now, by the lemma of $\$ 4$ of [5], we have

$$
\begin{aligned}
\left(1+|x|^{2}\right)|G(x)|\left|H^{(r)}(z-x)\right| & \leqq \frac{\alpha_{1} \alpha_{2}\left(1+|x|^{\imath}\right)\left(1+|x|^{2}\right)}{1+|z-x|^{t+2}} \\
& \leqq \frac{\alpha_{3}\left(1+|x|^{t+2}\right)}{1+|z-x|^{t+2}} \\
& \leqq \alpha_{4}\left(1+|z|^{t+2}\right)
\end{aligned}
$$

for all $b \leqq I(z) \leqq d$, where $\alpha_{3}, \alpha_{4}$ are some constants. Thus, we can find a constant $\alpha_{5}$ such that, for all $b \leqq g(z) \leqq d$,

$$
|K(z)| \leqq \alpha_{5}\left(1+|z|^{t+2}\right) \text {. }
$$

This implies immediately that $K$ has property $L[-\infty, a]$.

Corollary. Let $a, b$ be positive numbers and let $f \in \mathcal{S}$. Suppose that, for any $k,\left(d^{k} f / d X^{k}\right)(x)=O\left(e^{-b x}\right)$ as $x \rightarrow+\infty$ and that $\left(d^{k} f / d X^{k}\right)(x)=O\left(e^{a x}\right)$ as $x \rightarrow-\infty$. Then, for any $T \in s^{\prime}$, the Fourier transform of $f T$ can be represented by $\mathrm{e}(K)$ where $K$ satisfies property $L[-a, b]$. 
Proof. Let $s^{-}$and $s^{+}$be non-negative valued functions in $\varepsilon$ such that 1. $s^{-}(x)+s^{+}(x)=1$ for all $x \in K$.

2. $s^{+}(x)=0$ for $x \leqq-1, s^{-}(x)=0$ for $x \geqq 1$.

Then the functions $s^{+} f$ satisfies the hypotheses of Proposition 7 so that the Fourier transform of $s^{+} f T$ can be represented as $\mathfrak{e}\left(K^{+}\right)$where $K^{+}$satisfies property $L[-\infty, b]$. A similar argument shows that the Fourier transform of $s^{-} f T$ can be represented as $\mathrm{e}\left(K^{-}\right)$where $K^{-}$satisfies property $L[-a, \infty]$. Since $f T=s^{-} f T+s^{+} f T$, the result follows with $K=K^{-}+K^{+}$.

THeOREм 2. Let $B$ be a space which satisfies property $M$, and let $T \in D^{\prime}$. Let $a<b$ be real numbers, and suppose that $\tau_{-i a} T$ and $\tau_{-i b} T$ are in $B$. Then there exists a function $K$ with the following properties:

$(\alpha) K$ satisfies property $L[a, b]$.

( $\beta$ ) For each $c$ in $(1), \mathfrak{e}\left(\tau_{-c} K\right)=\tau_{-c} T$.

$(\gamma)$ Whenever $a<\mathfrak{g}(c)<b, \mathrm{e}\left(\tau_{-c} K\right) \in B$.

( $\delta)\left\{\mathfrak{e}\left(\tau_{-c} K\right)\right\}$ is bounded in $\boldsymbol{B}$ whenever $c$ stays in a compact set of (1).

Proof. Denote by $U$ the inverse Fourier transform of $T$; our hypotheses imply that $\exp (-a \cdot) U$ and $\exp (-b \cdot) U$ are in $B$. Let $t^{+}$and $t^{-}$be real valued, non negative functions in $E$ which satisfy

1. $t^{+}(x)+t^{-}(x)=1$ for all $x \in R$.

2. $t^{+}(x)=1$ for $x \geqq 1, t^{-}(x)=1$ for $x \leqq-1$.

Suppose that $a \leqq g(d) \leqq b$, then we have

$$
\begin{aligned}
\exp (i d \cdot) U & =t^{+} \exp (i d \cdot) U+t^{-} \exp (i d \cdot) U \\
& =t^{-} \frac{\exp (i d \cdot)}{\exp (-b \cdot)} \exp (-b \cdot) U+t^{+} \frac{\exp (i d \cdot)}{\exp (-a \cdot)} \exp (-a \cdot) U .
\end{aligned}
$$

If $a<g(d)<b$, then $t \exp (i d \cdot) / \exp (-b \cdot)$ and $t^{+} \exp (i d \cdot) / \exp (-a \cdot)$ are clearly in $S$. Thus, $\exp (i d \cdot) U \in \beta$ which means that $\tau_{-d} T \in B$.

On the other hand, if $a<g(d)<b$, then $t^{-} \exp (i d \cdot) / \exp (-b \cdot)$ vanishes for all $x \geqq 1$ and is $O[\exp (b-I(d) \cdot)]$ as $x \rightarrow-\infty$. Thus, by Proposition 7 , the Fourier transform of $t^{-}(\exp (i d \cdot) / \exp (-b \cdot)) \exp (-b \cdot) U$ can be represented as $\mathfrak{e}\left(K^{-}\right)$where $K^{-}$satisfies property $L[\mathfrak{g}(d)-b, \infty]$. Similarly, the Fourier transform of $t^{+}(\exp (i d \cdot) / \exp (-a \cdot)) \exp (-a \cdot) U$ can be represented as $\mathfrak{e}\left(K^{+}\right)$where $K^{+}$has property $L[-\infty, \mathfrak{g}(d)-a]$. We conclude from (6) that $\tau_{-d} T$ can be represented as $\mathrm{e}\left(K^{*}\right)$ where $K^{*}$ satisfies property $L[\mathfrak{g}(d)-b, \mathfrak{g}(d)-a]$. Combining the above with Theorem 1 , we see that we have established assertions $(\alpha),(\beta)$, and $(\gamma)$ of Theorem 2 .

Now, it is clear that

$$
\left\{t^{-} \frac{\exp (i d \cdot)}{\exp (-b \cdot)}\right\} \text { and }\left\{t^{+} \frac{\exp (i d \cdot)}{\exp (-a \cdot)}\right\}
$$

are bounded sets in $S$ whenever $d$ stays in a compact set of (1). Thus, on ac- 
count of the continuity of the map $f \rightarrow f V$ for $V \in B$ of $s \rightarrow B$, it follows from (6) that $\{\exp (i d \cdot) U\}$ is bounded in $B$ for $d$ in any compact set of (1). Part $(\delta)$ of Theorem 2 results immediately.

CoRollary. Let $a<b$ be real numbers, and let $F$ have property $L[a, b]$. Suppose that the boundary values of $F$ at $a$ and $b$ are in a space $B$ which satisfies property $M$. Then for any $z$ in the strip (1), $\mathrm{e}\left(\tau_{-z} F\right) \in B$. Moreover, if $K$ is any compact subset of (1), $\left\{\mathrm{e}\left(\tau_{-z} F\right)\right\}_{z \in K}$ is bounded in $B$.

Proof. Denote by $\tau_{-a} T$ the boundary value of $F$ at $a$ (where $T \in D^{\prime}$ ). Then, by Proposition 4, for any $z$ in (1), $\tau_{-z} T=\mathfrak{e}\left(\tau_{-z} F\right)$. This implies that $\tau_{-b} T$ is the boundary value of $T$ at $b$. The result is now an immediate consequence of Theorem 2.

We do not know whether, under the hypotheses of Theorem 2, the set $\left\{\tau_{-i c} T\right\}_{a \leqq c \leqq}$ is bounded in $B$. It is easy to give additional hypotheses on $B$ in order that this be true. In case that $B$ is a space $\mathfrak{L}_{p}$, we can obtain this result and even more precise information. However, we do not know, even for these spaces, the best possible result.

Fyor any $f \in L_{p}$, we denote by $\|f\|_{p}$ the usual norm of $f$ in $L_{p}$. For $S \in e L_{p}$, $\|S\|_{p}$ is defined as $\left\|e^{-1} S\right\|_{p}$ with a similar convention for $S \in \mathfrak{j} L_{p}$ or $S \in \mathfrak{L}_{p}$.

THEOREM 3. Under the hypotheses of Theorem 2, if $B$ is a space $\mathfrak{L}_{p}$, then, whenever $a<d<b$,

$$
\left\|\tau_{-i d} T\right\|_{p} \leqq\left\|\tau_{-i a} T\right\|_{p}+\left\|\tau_{-i b} T\right\|_{p} .
$$

In particular, $\left\{\tau_{-i c} T\right\}_{a \leqq c \leqq b}$ is bounded in $B$.

Proof. Let $a<d<b$, and denote by $U$ the inverse Fourier transform of $T$. Then, in Equation (6), we may clearly choose the functions $t^{+}$and $t^{-}$in such a way that

$$
\max _{x \in R} t^{-}(x) e^{(b-d)} \leqq 1, \quad \max _{x \in R} t^{+}(x) e^{(a-d) x} \leqq 1 .
$$

Now, it is obvious from the definitions that, for $V \in \mathfrak{i} L_{p}, F \in \mathcal{S}$,

$$
\|F V\|_{p} \leqq\|F\|_{\infty}\|V\|_{p} .
$$

Equation (7) results from this and Equation (6).

Regarding the spaces $L_{p}$, we have the following:

Theorem 4. Let $\bar{F}$ satisfy property $L[a, b]$ where $a<b$ are real numbers. Suppose that $\tau_{-i a} F$ and $\tau_{-i b} F \in L_{p}$, and moreover, that for some $\epsilon>0$ the set

$$
\left.\exp \left[-\cosh \pi\left(\frac{2}{b-a}-\epsilon\right)\left(z-\frac{b+a}{2}\right)\right] \mathfrak{e} \tau_{-z} F\right\}_{a \leqq} \mathcal{J}_{(z) \leqq b}
$$

is weakly bounded in $D^{\prime}$. Then $\log \left\|\tau_{-i x} F\right\|_{p}$ is a convex function of $c$ for $a \leqq c \leqq b$. 
Proof. Let $a \leqq d \leqq b$, and let $I I \in D$ satisfy $\|H\|_{p^{\prime}} \leqq 1$ where $1 / p+1 / p^{\prime}=1$. Set

$$
G(z)=\int_{-\infty}^{\infty} H(x) F(x+z) d x=\mathfrak{e} \tau_{-z} \bar{F} \cdot H=\tau_{-z} e \bar{F} \cdot H
$$

for $a \leqq g(z) \leqq b$. By Theorem 1 of $\S 2, G$ is an entire function. By our hypotheses, $G$ is bounded on the lines $\mathscr{G}(z)=a$ and $\mathscr{g}(z)=b$ by $\left\|\tau_{-i a} F\right\|_{p}$ and $\left\|\tau_{-i b} F\right\|_{p}$ respectively. In addition for some $\epsilon>0$,

$$
|\exp [-\cosh \pi(2 /(b-a)-\epsilon)(z-(b+a) / 2)] G(z)|
$$

is bounded in the strip $a \leqq \mathfrak{g}(z) \leqq b$. Thus, by the classical Phragmen-Lindelöf Theorem, we have

$$
\left|e \tau_{-i d} F \cdot H\right|=G(i d) \leqq \max \left(\left\|\tau_{-i a} F\right\|_{p},\left\|\tau_{-i b} F\right\|_{p}\right) .
$$

Since $D$ is dense in $L_{p^{\prime}}$, this gives

$$
\left\|\tau_{-i d} F\right\|_{p} \leqq \max \left(\left\|\tau_{-i a} F\right\|_{p},\left\|\tau_{-i b} F\right\|_{p}\right) .
$$

Now, let $a \leqq a^{\prime} \leqq d \leqq b^{\prime} \leqq b$, and choose $\alpha$ so that $e^{\alpha a^{\prime}}\left\|\tau_{-i a^{\prime}} F\right\|_{p}=c^{\alpha b^{\prime}}\left\|\tau_{-\imath b^{\prime}} F\right\|_{p}$. Then, applying the inequality (10) to $\exp (-i \alpha) F$ in place of $F$, we obtain

$$
e^{\alpha d}\left\|\tau_{-i d} F\right\|_{p} \leqq e^{\alpha a^{\prime}}\left\|\tau_{-i a^{\prime}} F\right\|_{p}=e^{\alpha b^{\prime}}\left\|\tau_{-i b^{\prime}} F\right\|_{p} .
$$

Using the fact that $\alpha=\left(\log \left\|\tau_{-i a^{\prime}} F\right\|_{p}-\log \left\|\tau_{-i b^{\prime}} F\right\|_{p}\right) /\left(b^{\prime}-a^{\prime}\right)$ we find easily

$$
\log \left\|\tau_{-i d} F\right\|_{p} \leqq \frac{b^{\prime}-d}{b^{\prime}-a^{\prime}} \log \left\|\tau_{-i a^{\prime}} F\right\|_{p}+\frac{d-a^{\prime}}{b^{\prime}-a^{\prime}} \log \left\|\tau_{-i b^{\prime}} F\right\|_{p}
$$

which is the desired result.

REMARK 1 . It is probably always true that if $\bar{F}$ satisfies property $L[a, b]$ then, for some $\epsilon>0$,

$$
\left\{\exp \left[\cosh \pi\left(\frac{2}{b-a}-\epsilon\right)\left(z-\frac{b+a}{2}\right)\right] \mathfrak{e} \tau_{-z} F\right\}_{a \leq \mathcal{I}(z) \leqq b}
$$

is weakly bounded in $D^{\prime}$.

REMARK 2 . In the above theorem we can replace the spaces $L_{p}$ by any normed space $A$ of functions on $R$ such that

(1) $A$ satisfies property $M$.

(2) $e D$ is dense in $A^{\prime}$.

(3) For any $f \in A, x$ a real number, the $A$-norm of $\tau_{i x} f$ is the same as the $A$-norm of $f$.

In particular, the theorem is valid for the spaces $\mathfrak{L}_{p}$ and, by Theorem 3 , the additional hypothesis (9) is not needed for these spaces.

THEOREM 5. Let a be a real number, and let $B$ be a space which satisfies property $M$. Let $T \in D^{\prime}$, and suppose that, for some $d>0,\left\{\exp (-d c) \tau_{-i c} T\right\}_{c \geqq a}$ 
is weakly bounded in $D^{\prime}$. Suppose, moreover, that $\tau_{-i a} T \in M$. Then there exists a function $\bar{F}$ satisfying property $L[a, \infty]$ such that

$(\alpha)$ Whenever $\mathfrak{g}(z) \geqq a, \mathfrak{e}\left(\tau_{-z} F\right)=\tau_{-z} T$.

( $\beta$ ) For all $z$ with $g(z) \geqq a, \mathfrak{e}\left(\tau_{-z} F\right) \in B$.

$(\gamma) \mathfrak{e}\left(\tau_{-z} F\right)$ is bounded in $B$ when $z$ stays in a compact set of $g(z)>a$.

Proof. Denote by $U$ the inverse Fourier transform of $T$, so that $\tau_{d} U$ is the inverse Fourier transform of $\exp (i d) T$. Since $\left\{\tau_{-i c}[\exp (i d \cdot) T]\right\}_{c \geq a}$ is weakly bounded in $D^{\prime}$, it follows from Theorem 4 of $\$ 2$ that the carrier of $U$ is contained in $[-d \leqq x<\infty]$.

Now, if $\mathscr{g}(z) \geqq a$, the inverse Fourier transform of $\tau_{-z} T$ is

$$
F^{-1}\left(\tau_{-2} T\right)=\exp (i z) U=t \frac{\exp (i z \cdot)}{\exp (i a \cdot)} \exp (i a) U
$$

where $t$ is a real-valued indefinitely differentiable function on $R$ such that $t(x)=1$ for $x \geqq-d, t(x)=0$ for $x \leqq-d-1$. Now, clearly, for $\mathscr{g}(z)>a$,

$$
t \exp (i z \cdot) / \exp (i a \cdot) \in S,
$$

and, in fact, the functions $t \exp (i z \cdot) / \exp (i a \cdot)$ form a bounded set in $S$ whenever $z$ lies in a compact set of $g(z)>a$. The result now follows by the definition of property $M$.

Corollary. Let $F$ be an entire function of exponential type, and let $\boldsymbol{B}$ be a space which satisfies property $M$. Suppose for some $d, e \tau_{-i d} \bar{F} \in B$. Then for every $z, \mathrm{e}\left(\tau_{i z} \bar{F}\right) \in B$. Moreover, as $z$ varies in a compact set, $\left\{\mathrm{e} \tau_{i z} \bar{F}\right\}$ remains in a compact set of $B$.

Proof. Since $B \subset \mathcal{S}^{\prime}$, by the Paley-Wiener theorem (see $[5 ; 7]$ ) we see that the Fourier transform $U$ of $e \bar{F}$ is a distribution of compact carrier. Suppose that the carrier of $U$ is contained in the interval $[-a \leqq x \leqq a]$. Then the sets $\left\{\exp (-(a+1) c) \tau_{-i c} e \bar{F}\right\}_{c \geq-1}$ and $\left\{\exp \left((a+1 c) \tau_{-i c} c \bar{F}\right\}_{c \leq 1}\right.$ are bounded in $D^{\prime}$. The result now follows easily from Theorem 5 .

8. General remarks. Theorem 3 of $\S 3$ shows us that

$$
\left\|\tau_{-i d} T\right\|_{p} \leqq\left\|\tau_{-i a} T\right\|_{p}+\left\|\tau_{-i b} T\right\|_{p}
$$

in the notation of that theorem. The question naturally arises as to the best possible (smallest) expression for $\left\|\tau_{-i d} T\right\|_{p}$ in terms of $\left\|\tau_{-i a} T\right\|_{p}$ and $\left\|\tau_{-i b} T\right\|_{p}$. For this purpose, we may assume (by considering $\tau_{-i d} T$ instead of $T$ ) that $d=0$ and that $a=-c, c>0$. In that case, in virtue of Equation (6) of $\$ 7$, the best possible result which can be obtained by our methods depends on the solution of the following problem: Among all functions $\lambda \in \mathcal{E}$ for which, for any $k$,

$$
\left[\frac{d^{k}}{d X^{k}}(\lambda \exp (b \cdot))\right](x)=O\left(e^{-b x}\right) \text { as } x \rightarrow+\infty,
$$




$$
\left[\frac{d^{k}}{d X^{k}}(\lambda \exp (b \cdot))\right](x)=O\left(e^{c x}\right) \text { as } x \rightarrow-\infty,
$$

with a similar property for $(1-\lambda) \exp (a \cdot)$ in place of $\lambda \exp (b \cdot)$, determine the minimum value of the expression

$$
\alpha_{1} \max _{x \in R}|\lambda(x) \exp (b x)|+\alpha_{2} \max _{x \in R}|(1-\lambda(x)) \exp (a x)|
$$

where $\alpha_{1}$ and $\alpha_{2}$ are constants. (For the application to our problem of the best possible result, we take $\alpha_{1}=\left\|\tau_{-i a} T\right\|_{p}, \alpha_{2}=\left\|\tau_{-i b} T\right\|_{p}$.) We have not yet been able to solve the above minimum problem.

In the corollary to Theorem 2 of $\S 3$, we assumed that $F$ satisfies property $L[a, b]$. Now, this "growth at infinity" property of $F$ is certainly not the best possible. In order to improve this, we must make use of the following considerations:

Let $V$ be a topological vector space of entire functions such that

1. For each $G \in V, z \in C, \tau_{z} G \in S$ and $\tau_{z} G \in V$.

2. For any $T \in V^{\prime}, z \rightarrow \tau_{z} T$ is a continuous map of $C$ into $V^{\prime}$.

3. For any real number $t$ and any $G \in V$, exp (it.) $G$ is again in $V$.

4. There is no $z \in C$ at which all functions of $V$ vanish.

Now, if $H$ is any locally summable function on $R$ such that, for any $G \in V$, $\int \bar{H}(x) G(x) d x$ is defined and also $G \rightarrow \int \bar{H}(x) G(x) d x$ is a continuous linear function on $V$, then we define $e_{V} H \in V^{\prime}$ by

$$
\mathfrak{e}_{V} H \cdot G=\int \bar{H}(x) G(x) d x
$$

for any $G \in V$. If $a<b$, we can now define a property $L_{V}[a, b]$ in a method analogous to the definition of property $L[a, b]$ (which we might now regard as property $L_{D}[a, b]$.) We may also define the concept of the boundary value, over $V$, of a function $K$ at $a$ in a manner analogous to the definition of boundary value in $\S 3$. For each result of $\S 3$, the analogous result for the space $V$ instead of $D$ may now be proven exactly as the corresponding result of $\S 3$ was proven. In particular, the Corollary to Theorem 2 of $\$ 3$ goes over into the following result which is the best possible obtainable by our methods.

Let $B$ be a space which satisfies property $M$, and $V$ a space which satisfies Conditions $1,2,3,4$ above. Let $a<b$, and let $F$ satisfy property $L_{V}[a, b]$. Suppose that the boundary values over $V$ of $F$ at $a$ and $b$ are in $B$. Then, for any $z$ such that $a<g(z)<b, e_{V}\left(\tau_{-z} F\right) \in B$ and, if $K$ is a compact set of the strip $a<\mathfrak{g}(z)<b$,

$$
\left\{e_{V}\left(\tau_{-z} F\right)\right\}_{z \in K}
$$

is bounded in $\boldsymbol{B}$.

From the above, it follows that $F$ satisfies the conclusions of the above if, 
for example, there exists a constant $B$ such that, for each closed substrip $W$ of $a<g(z)<b$, we can find a constant $A$ with

$$
|F(z)| \leqq A e^{|z|^{B}}
$$

for all $z \in W:$ For in that case, let $k$ be an even integer greater than $B$; let $V$ be the space of all multiples of $\exp \left(-Z^{k}\right)$ by functions of the form $P \exp (i t \cdot)$ where $P$ is a polynomial and $t$ is a real number. The topology of $V$ is defined by means of the semi-norms

$$
v_{d, p}(G)=\max _{z \in C_{d}}\left|e^{z p} G(z)\right|
$$

for any $G \in V$, where $d$ is any positive number, and $0<p<k$. It is easily seen that $V$ satisfies Conditions $1,2,3,4$ above and that $F$ satisfies property $L_{V}[a, b]$.

It is possible to improve the above method slightly by considering topological vector spaces $W$ of functions which are analytic in a strip $\alpha<\mathfrak{g}(z)<\beta$ and which satisfy properties analogous to Properties 1, 2, 3, 4 above. Of course, for $z \in C, H \in W, \tau_{z} H$ need no longer be in $W$, so Conditions 1 and 2 have to be suitably modified. However, we shall omit the details of this method.

\section{BIBLIOGRAPHY}

1. N. Bourbaki, Intégration, vol. 13, Livre VI, Chapitre III, Paris, 1953.

2. J. Dieudonné and L. Schwartz, La duality dans les espaces (F) et (\&F), Ann. Inst. Fourier, Grenoble, vol. 1 (1950) pp. 61-101.

3. L. Ehrenpreis, Theory of distributions in locally compact spaces, Part I, Columbia University thesis, 1953; Memoirs Amer. Math. Soc., no. 21, 1956.

4. - Mean periodic functions I, Amer. J. Math., vol. 77 (1955) pp. 293-328

5. - Analytic functions and the Fourier transform of distributions I, Ann. of Math., vol. 63 (1956) pp. 129-159.

6. E. Hille, Functional analysis and semi-groups, New York, 1948.

7. L. Schwartz, Théorie des distributions, vol. I and II, Paris, Hermann and Company, $1951,1952$.

8. E. C. Titchmarsh, The theory of functions, Oxford, 1930.

9. - Theory of Fourier integrals, Oxford, 1937.

BrandeIs UNIVERSITY, WaLTHAM, Mass.

Johns Hopkins University, BALTimore, MD. 